\title{
Atom- and field-state evolution in the Jaynes-Cummings model for large initial fields
}

\author{
Julio Gea-Banacloche \\ Department of Physics, University of Arkansas, Fayetteville, Arkansas, 72701 \\ and Instituto de Optica, Consejo Superior de Investigaciones Científicas, Serrano 121, 28006 Madrid, Spain
}

(Received 19 March 1991)

\begin{abstract}
An asymptotic result is derived for the Jaynes-Cummings model of a two-level atom interacting with a quantized single-mode field, which is valid when the field is initially in a coherent state with a large average photon number. It is shown that for certain initial atomic states the joint atom-field wave function factors into an atomic and a field part throughout the interaction, so that each system remains separately in a pure state. The atomic part of the wave function displays a crossing of trajectories in the atom Hilbert space that leads to a unique state for the atom, independent of its initial state, at a specific time $t_{0}$ (equal to half the revival time). The field part of the wave function resembles a crescent squeezed state. The well-known collapses and revivals are investigated from this perspective. The collapse appears to be associated with a "measurement" of the initial state of the atom with the field as the measuring apparatus. The measurement is not complete for finite average photon number: the system is instead left in a coherent superposition of macroscopically distinct states. At the half-revival time $t_{0}$ this superposition of states is entirely in the field part of the state vector, so that the (pure) state of the field at that time is of the form sometimes referred to as a "Schrödinger cat." The revivals of the population inversion are seen to be entirely due to the fact that the linear superposition of the two macroscopically distinct field states is coherent (i.e., a pure state), as opposed to an incoherent mixture.
\end{abstract}

PACS number(s): 42.50. $-\mathrm{p}, 03.65 . \mathrm{Bz}, 42.52 .+\mathrm{x}$

\section{INTRODUCTION AND OVERVIEW}

The Jaynes-Cummings model (JCM) [1] is one of the simplest of quantum-electrodynamical systems: a twolevel atom interacting with a single mode of the quantized radiation field, in the so-called rotating-wave approximation (RWA). In addition to its being exactly solvable, it has become increasingly well approximated by recent experiments involving the passage of single atoms through superconducting microwave cavities [2]. In spite of its apparent simplicity, it has provided theorists with many nontrivial and unexpected results through the years, beginning with the well-known phenomena of collapses and revivals of the atomic population inversion $[3,4]$. Much of the recent research has focused on the squeezing of the field predicted by the model $[5,6]$.

One of the most interesting features of the JCM is that it involves two systems in interaction, with every feature of the interaction and of each system described quantum mechanically: there are no external, $c$-number-type forces or potentials. (In fact, one reason why the model was originally introduced [1] was to find out in which way the quantization of the field affected the predictions for the evolution of the atom, that is, to compare with the semiclassical theory, where the field is not treated as a quantized variable.) It is therefore possible to use the JCM to investigate many issues of interest involving interacting quantum systems, quantum correlations and entanglement, and perhaps even state preparation and measurement. Especially noteworthy is the fact that one of the two systems, namely, the field, has a well-defined classical limit, which is usually taken to be a Glauber coherent state [7] with a very large number of photons.
Not many previous studies have emphasized this aspect of the JCM $[8,9]$. Among them, as especially relevant to the present paper, one must note the research of Phoenix and Knight [10], who used the entropy to study the correlations between the field and atom as well as the "purity" of the state of each. They showed that the field in the JCM was essentially a two-state quantity [11]. Their numerical calculations with states with nottoo-large numbers of photons also show that the field (and hence the atom) most closely returns to a pure state somewhere within the so-called "collapse region."

It was recently shown by the present author [12] that for a large average number of photons, if the initial state of the field is a coherent state, the states of the atom and field become in fact arbitrarily pure at a specific time $t_{0}$ equal to one-half the conventionally defined "revival time" $t_{R}$; that is, the atom and field spontaneously become disentangled at this time. Moreover, it was found that the state of the atom at the time $t_{0}$ is completely independent of the initial atomic state. One of the purposes of the present paper is to investigate the consequences of this result, as well as to present a more detailed and careful proof than could be given in Ref. [12]; this is done in Sec. II and Appendix A, respectively.

It turns out that for large numbers of photons the most convenient basis of atomic states is not provided by the energy eigenstates but by special states $|+\rangle$ and $|-\rangle$, defined in Sec. II, which are eigenstates of the semiclassical interaction Hamiltonian (assuming, as will be done throughout this paper, exact resonance between the atom and the field). These states, as shown in this paper, have the remarkable property that if the atomic system is initially prepared in one of them, the state vector for the 
atom-field system at later times factors out, to a good approximation, into an atom part and a field part; that is, the two systems do not become entangled but remain each in an approximately pure state. In spite of this property, however, the evolution of each (atomic or field) part of the wave function under these circumstances is decidedly nontrivial, and in particular nonunitary, in the sense that initially orthogonal atomic states do not remain orthogonal in the course of their evolution. (The total wave function for the joint atom-field system does evolve unitarily, of course, since this is taken to be a closed system.) The result is like a "crossing of trajectories" in the atom Hilbert space at $t=t_{0}$. This is discussed and explored in Sec. II.

The field part of the wave function that evolves when the initial atomic state is one of the states $|+\rangle$ or $|-\rangle$ also has interesting properties, which are studied in some detail in Appendix B; it corresponds to a field state which (always in the limit of very large number of photons) has a large amplitude and becomes squeezed, as time passes, in a way analogous to the "crescent states" discussed by Yamamoto and co-workers $[13,14]$. The field state corresponding to an initial atomic state $|+\rangle(|-\rangle)$ has a macroscopically well-defined phase which grows (decreases) with time; in the field's phase space the two states are represented by counterrotating phasors. This phenomenon underlies the recent observations of Eiselt and Risken [15,16] (see also Refs. [17] and [18]) regarding the quasiprobability distribution for the field in the JCM when the atom is prepared in a state (such as the energy eigenstates) which is a linear superposition of $|+\rangle$ and $|-\rangle$. When this fact is taken together with the observation that all initial atomic states lead to the same atomic state as $t_{0}$, it follows that the state of the field at this time, for an initial atomic energy eigenstate, is in fact a coherent linear superposition of macroscopically distinct states (states of opposite phase); such a superposition has been dubbed a "Schrödinger cat" [19]. It is analyzed in Sec. IV, where it is shown that the signature of the mutual coherence between the two macroscopically distinct parts of the field wave function at the time $t_{0}$ is none other than the well-known revival of the population inversion at the later time $t_{R}$.

Section III deals with another interesting question. As said above, the two orthogonal initial atomic states $|+\rangle$ and $|-\rangle$ lead to two field states which become in time macroscopically distinct. Hence, information about the initial state of the atom becomes stored in the field, which is a potentially macroscopic system with a classical limit. Does this mean that a measurement of the initial state of the atom has been carried out? The analysis in Sec. III points to a number of interesting analogies between this process and some aspects of the quantum theory of measurement; it is shown, for instance, that at the time of the well-known JCM collapse the reduced density operator for the atom becomes diagonal in the basis of the states $|+\rangle$ and $|-\rangle$, and that this collapse takes place as soon as the two field states associated with $|+\rangle$ and $|-\rangle$ become macroscopically distinguishable. Whether it is consistent or not to postulate that a "collapse of the wave function," in the sense of quantum measurement theory, takes place at the traditional collapse time is a question dealt with briefly in Sec. III also. In any case, for a finite number of photons in the field it is clear that one could have at most a sort of "incomplete measurement," that is, the total system is left in a coherent superposition of macroscopically distinct states. The spontaneous disentanglement of atom and field that takes place at $t_{0}$ causes this superposition to affect only the field part of the state vector at this time, which results in the Schrödinger cat discussed in Sec. IV.

In addition to the results that have been summarized above, the present work brings together, in a special limit, many observations made by a large number of people over the years, showing how in this limit all these observations may be derived from a simple expression for the atom and field evolution. It is quite possible that the results of this paper might lead to further insights in other aspects of the JCM not covered here, as well as in the general study of the dynamical and coherence properties of "open" quantum systems, and possibly also in quantum measurement theory. A brief outlook on some possible directions in which further research might proceed is presented in Sec. V.

\section{NONUNITARY, QUASI-PURE-STATE EVOLUTION IN THE JCM}

\section{A. General results}

This section introduces the JCM and presents the main analytical result of the paper. The JCM evolution turns out to be very simple, for large average number of photons, if one looks at special initial states of the atom, corresponding to well-defined values of the dipole moment amplitude. The evolution of any other initial state may be understood as a linear superposition of these.

The JCM involves only two interacting quantum systems: a two-level atom, whose upper and lower states may be written, respectively, as $|a\rangle$ and $|b\rangle$, and a single mode of the quantized electromagnetic field, whose annihilation and creation operators are denoted by $a$ and $a^{\dagger}$. Assuming exact resonance, and performing the rotating-wave approximation, the interaction Hamiltonian adopts the simple form

$$
H_{I}=\hbar g\left(|a\rangle\left\langle b\left|a+a^{\dagger}\right| b\right\rangle\langle a|\right),
$$

where $g=d\left(\omega / \hbar V \epsilon_{0}\right)^{1 / 2}$ is a coupling constant ( $d$ is the atomic dipole matrix element for the transition, $\omega$ is the transition frequency, and $V$ the mode volume).

The exact solution for an initial atomic state $|\psi(0)\rangle_{\text {atom }}=\alpha|a\rangle+\beta|b\rangle$ and field state $|\psi(0)\rangle_{\text {field }}$ $=\sum_{n=0}^{\infty} C_{n}|n\rangle$ is

$$
\begin{aligned}
|\psi(t)\rangle=\sum_{n=0}^{\infty}\{[ & \alpha C_{n} \cos (g \sqrt{n+1} t) \\
& \left.\quad-i \beta C_{n+1} \sin (g \sqrt{n+1} t)\right]|a\rangle \\
+ & {\left[-i \alpha C_{n-1} \sin (g \sqrt{n} t)\right.} \\
& \left.\left.+\beta C_{n} \cos (g \sqrt{n} t)\right]|b\rangle\right\}|n\rangle .
\end{aligned}
$$


In general the state described by (2) is a highly entangled state of the field and the atom. For an initial coherent state field with large photon number, however, the result simplifies considerably if one looks at the evolution of the initial atomic states $|+\rangle$ and $|-\rangle$, defined by

$$
| \pm\rangle=\frac{1}{\sqrt{2}}\left(e^{-i \phi}|a\rangle \pm|b\rangle\right)
$$

where $\phi$ is the phase of the initial field coherent state $|v\rangle$ :

$$
|\psi(0)\rangle_{\text {field }}=|v\rangle \equiv e^{-|v|^{2} / 2} \sum_{n=0}^{\infty} \frac{|v|^{n}}{\sqrt{n !}} e^{-i n \phi}|n\rangle
$$

(the average number of photons is clearly $\bar{n}=|v|^{2}$ ).

Formally, what is special about the states $| \pm\rangle$ is that they are eigenstates of the "semiclassical (sc) Hamiltonian" which is obtained when replacing the operators $a\left(a^{\dagger}\right)$ in (1) by the $c$-number field amplitude $v\left(v^{*}\right)$ :

$$
H_{I_{\mathrm{sc}}}=\hbar g\left(|a\rangle\left\langle b\left|v+v^{*}\right| b\right\rangle\langle a|\right) .
$$

Therefore, in the classical limit, these states do not really evolve at all (except for an overall phase). With a quantized field, as in the Hamiltonian (1), they are found indeed to evolve, but in a way which, in the limit of large $\bar{n}$, is very simple, as will be shown presently.

Physically, the states $| \pm\rangle$ correspond to well-defined values of the atomic dipole amplitude which are either in phase or $180^{\circ}$ out of phase with the applied field, which is the reason why, classically, no exchange of energy with the field takes place. The fact that these states do not evolve in the semiclassical theory was pointed out by Puri and Agarwal [20], who suggested that they could be used as sensitive probes of the differences between semiclassical or neoclassical and fully quantized theories [21]; Zaheer and Zubairy [22] referred to them as "trapping states" and studied the emission spectrum for an atom initially prepared in one of these states. [Other "trapping" states in the JCM for other (not coherent) initial field states have been studied in Ref. [23].]

This paper's main analytical result is the following expression for the time evolution of the atom-field system, when the initial state is $|+\rangle|v\rangle$ [with $|v\rangle$ given by (4)], in the limit as $\bar{n} \rightarrow \infty$ :

$$
\begin{aligned}
\left.|+\rangle|v\rangle\right|_{t=0} \rightarrow & \frac{1}{\sqrt{2}}\left(e^{-i \phi} e^{-i g t / 2 \sqrt{\bar{n}}}|a\rangle+|b\rangle\right) \\
& \times e^{-\bar{n} / 2} \sum_{n=0}^{\infty} \frac{\bar{n}^{n / 2}}{\sqrt{n !}} e^{-i n \phi} e^{-i g t \sqrt{n}}|n\rangle
\end{aligned}
$$

and the corresponding equation for the evolution of a system prepared in the initial state $|-\rangle|v\rangle$ :

$$
\begin{aligned}
\left.|-\rangle|v\rangle\right|_{t=0} \rightarrow & \frac{1}{\sqrt{2}}\left(e^{-i \phi} e^{i g t / 2 \sqrt{\bar{n}}}|a\rangle-|b\rangle\right) \\
& \times e^{-\bar{n} / 2} \sum_{n=0}^{\infty} \frac{\bar{n}^{n / 2}}{\sqrt{n !}} e^{-i n \phi} e^{i g t \sqrt{n}}|n\rangle .
\end{aligned}
$$

The proof of Eqs. (6) is found in Appendix A. The result is rigorous in the following sense: the difference between the exact solution (2) and the right-hand side of (6a) [or (6b)] is a vector whose norm vanishes in the limit $\bar{n} \rightarrow \infty$.
The result holds for any finite time, and even for $t \rightarrow \infty$, provided, as the derivation in Appendix A shows, that $t$ go to infinity slowly enough to have $t / \bar{n} \rightarrow 0$. This last provision is important, because, as one can see from Eq. (6), as $\bar{n} \rightarrow \infty$ the evolution of the atomic part of the wave function becomes "infinitely slow"; in particular, it is well known $[3,4]$ that the relevant time scale for the JCM revivals is $t_{R} \equiv 2 \pi \sqrt{\bar{n}} / g$. Since, however, $t_{R} / \bar{n} \rightarrow 0$ as $\bar{n} \rightarrow \infty$, the result (6) holds accurately over an arbitrarily large number of revivals, provided $\bar{n}$ is large enough.

In practice, for a finite time, the results in Appendix A may be used to estimate how large $\bar{n}$ has to be in order for the asymptotic solution to be a good approximation over that time. For atomic operators, whose expectation values are typically of the order of unity, the error in the expectation values calculated using the asymptotic approximation should not be greater than the norm of the terms neglected. In fact, numerical calculations (to be presented elsewhere) show this to be a rather conservative estimate, and that the asymptotic approximation is remarkably good over one revival time even for photon numbers as small as, say, $\bar{n}=25$ (compare also the results in Ref. [12] for $\bar{n}=49$ ). This is fortunate from the point of view of the possibility of eventually observing some of these results experimentally, which is being studied presently and will be discussed at length in a later publication. One may note in particular that for such low photon numbers the Rabi frequency $g \sqrt{\bar{n}}$ is typically much smaller than the atomic transition frequency $\omega$ (e.g., for the recent micromaser experiments of the Munich group [2], $g / 2 \pi=44 \mathrm{kHz}$ and $\omega / 2 \pi=21.5 \mathrm{GHz}$ ), which means that the RWA used to write the Hamiltonian (1) would be quite appropriate to describe the atomfield interaction (for a treatment of the atom-field interaction without the RWA, see Ref. [24]).

The main restriction to the usefulness of Eq. (6) stems from the fact that some of the terms neglected correspond to vectors whose norm squared may go to zero only as fast as $1 / \bar{n}$. If the state vector (6) is used to calculate expectation values of field operators, the result may not be quite reliable if for some reason the leading order in $\bar{n}$ vanishes; thus $\left\langle a^{2}\right\rangle$ and $\langle a\rangle$, for instance, will be correct to leading order in $\bar{n}$, but the difference $\left\langle a^{2}\right\rangle-\langle a\rangle^{2}$ may not be given correctly by (6) if the leading order in $\bar{n}$ cancels, as is the case when calculating squeezing. In spite of this, the vectors

$$
\left|\Phi_{ \pm}(t)\right\rangle=e^{-\bar{n} / 2} \sum_{n=0}^{\infty} \frac{\bar{n}^{n / 2}}{\sqrt{n !}} e^{-i n \phi} e^{\mp i g t \sqrt{n}}|n\rangle
$$

appearing in Eq. (6) are useful to predict, at least qualitatively, many properties of the field in the JCM in the large $\bar{n}$ limit. This is further discussed in Appendix B.

Since the states $|+\rangle$ and $|-\rangle$ form a basis set for the atom, the evolution of any other initial state can be expressed as a simple linear combination of (6a) and (6b). For instance, if the atom is initially in the excited state $|a\rangle=e^{i \phi}(|+\rangle+|-\rangle) / \sqrt{2}$ the total state vector evolves into 


$$
\begin{aligned}
\frac{1}{\sqrt{2}}\left(e^{-i g t / 2 \sqrt{\bar{n}}}|a\rangle+e^{i \phi}|b\rangle\right)\left|\Phi_{+}(t)\right\rangle \\
+\frac{1}{\sqrt{2}}\left(e^{i g t / 2 \sqrt{\bar{n}}}|a\rangle-e^{i \phi}|b\rangle\right)\left|\Phi_{-}(t)\right\rangle .
\end{aligned}
$$

As will be shown later, the field states $\left|\Phi_{ \pm}(t)\right\rangle$, given by Eq. (7), correspond to a macroscopic field rotating, in the phasor plane, clockwise and counterclockwise, respectively. Thus Eq. (8) exhibits the splitting of the field quasiprobability distribution found in Refs. [15-18]. This point will be discussed at length in the following sections.

Returning to the evolution equations (6), their most remarkable property is that the states appearing in them are product states [unlike, e.g., the general form (1) or the special case (8)]. This means that, in the limit of large $\bar{n}$ in which (6) is valid, both the field and the atom remain in pure states throughout the interaction if the atom is initially prepared in one of the states $| \pm\rangle$. Clearly, no other atomic states have this property.

Equation (6) implies that one can at all times assign a well-defined state to the atom prepared in one of the states $| \pm\rangle$; yet the evolution of such a state is nonunitary. This is seen in its most extreme instance at the special time $t_{0} \equiv t_{R} / 2=\pi \sqrt{\bar{n}} / \mathrm{g}$, where the atomic wave functions in (6a) and (6b) become, in fact, identical (except for an overall minus sign) and equal to

$$
\left|\psi_{0}\right\rangle=\frac{1}{\sqrt{2}}\left(-i e^{-i \phi}|a\rangle+|b\rangle\right)
$$

Thus atomic systems prepared initially in the orthogonal states $|+\rangle$ and $|-\rangle$ find themselves in the same atomic state at the time $t_{0}$, while remaining throughout their evolution, to a good approximation, in a pure state. This is in contrast with normal unitary evolution, which preserves orthogonality between states. Here neither the field nor the atom separately evolve unitarily, even though a state vector for each of them is well defined at all times. [The total wave function, naturally, does evolve unitarily, since the atom-field system is assumed to be closed. This means, in particular, that the orthogonality lost by the atomic state must be taken up by the field state at the time $t_{0}$, i.e., that the states $\left|\Phi_{+}\left(t_{0}\right)\right\rangle$ and $\left|\Phi_{-}\left(t_{0}\right)\right\rangle$ must be orthogonal, which, in the limit $\bar{n} \rightarrow \infty$, they are.]

Clearly, the nonunitarity arises from the fact that neither the atom nor the field alone is a closed system; the remarkable fact is that, in spite of this, a well-defined state vector for each of them exists at all times. One is tempted to consider an analogy with classical mechanics: there, the trajectories of a conservative system cannot cross, whereas for a dissipative system, on the other hand, it is quite common for trajectories to, e.g., converge to a point attractor (or to more complicated attractors). What the present example illustrates is an instance of "crossing of quantum-mechanical trajectories" for an open quantum system. The analogy is intriguing, and it might be worth investigating whether this kind of phenomenon might be common in open quantum systems in general.

One might think that the atomic "trajectories" that have the special states $|+\rangle$ and $|-\rangle$ as starting points are rather special themselves, i.e., far from generic. It will be shown in Sec. III that this may not necessarily be the case.

\section{B. Atomic-state preparation at $t=t_{0}$}

Since atomic systems prepared initially in either of the two basis states evolve towards the same state at $t=t_{0}$, this means that in every case, regardless of the initial atomic state, at the time $t=t_{0}$ the atom will be in a pure state, and that state, independent of the initial conditions, is $\left|\psi_{0}\right\rangle$ given by (9). This was recently pointed out by the present author [12]. (As mentioned in the Introduction, Phoenix and Knight [10] had previously noticed, in calculations involving not-too-large values of $\bar{n}$, that if the initial states of the atom and field were both pure, they were both closer to pure again around the time $t_{0}$.)

This means that the JCM could be used to prepare a specific atomic state, at a given time, in a way which is completely independent of what the initial state of the atom might be. It is perhaps worth emphasizing the novelty of this result. Semiclassically, a state such as $\left|\psi_{0}\right\rangle$ could be prepared by a $\pi / 2$ pulse, beginning with an atom in the ground state; but if the atom is initially in the upper state instead, the same $\pi / 2$ pulse would yield not the state $\left|\psi_{0}\right\rangle$ but one orthogonal to it (by unitarity). For the quantized-field system considered here, however, the ground and excited states, as well as any linear combination of them, all evolve towards $\left|\psi_{0}\right\rangle$ at $t=t_{0}$.

In fact, the state of the atom at $t=t_{0}$ is the pure state $\left|\psi_{0}\right\rangle$ even if initially the atom was not prepared in a pure state at all. Suppose in fact that the initial atomic state is described by a density operator $\rho_{\mathrm{at}}(0)$, which, without any loss of generality, may be taken to be diagonal in some basis $\left\{\left|\psi_{1}\right\rangle,\left|\psi_{2}\right\rangle\right\}$. Then, the total initial state for the system will be

$\rho_{\text {tot }}(0)=\rho_{11}\left|\psi_{1}\right\rangle|v\rangle\left\langle v\left|\left\langle\psi_{1}\left|+\rho_{22}\right| \psi_{2}\right\rangle\right| v\right\rangle\langle v|\left\langle\psi_{2}\right|$.

But, since the states $\left|\psi_{1}\right\rangle|v\rangle$ and $\left|\psi_{2}\right\rangle|v\rangle$ must evolve, respectively, toward states $\left|\psi_{0}\right\rangle\left|\phi_{1}\right\rangle$ and $\left|\psi_{0}\right\rangle\left|\phi_{2}\right\rangle$ at time $t_{0}$ (where $\left|\phi_{1}\right\rangle$ and $\left|\phi_{2}\right\rangle$ are some orthogonal field states), the total state for the system at $t_{0}$ will be

$\rho_{\text {tot }}\left(t_{0}\right)=\left|\psi_{1}\right\rangle\left\langle\psi_{0}\right| \otimes\left(\rho_{11}\left|\phi_{1}\right\rangle\left\langle\phi_{1}\left|+\rho_{22}\right| \phi_{2}\right\rangle\left\langle\phi_{2}\right|\right)$,

which again shows the atom to be in the pure state $\left|\psi_{0}\right\rangle$, and the field, incidentally, in a mixed state. In terms of entropy, one could say that all the entropy of the atom at the time $t=0$ is transferred to the field at $t=t_{0}$.

One can think of the following optical analogy. The polarization states of a photon may be described in a two-dimensional state space entirely analogous to that of the two-level atom. In terms of photons, the present device might be likened to an ideal polarizer that would transmit every photon with unit probability and, regardless of their initial polarization, place them all in the same polarization state upon transmission. No such device exists for photons, yet the present study shows how for a two-level atom one could conceivably be built.

Note also that this hypothetical ideal polarizer would work even if the initial photon did not have a definite po- 
larization at all; for instance, if it were a member of a pair in an entangled polarization state, such as those used in the experimental tests of the Bell inequalities [25]. For the atom in the JCM, this is also easy to verify explicitly from the results presented so far: Even if at $t=0$ the atom is an entangled state with some other system, at the time $t_{0}$ it is in a pure, nonentangled state; all the entanglement is transferred to the field.

Consider, for instance, the evolution of the initial state

$$
|e(0)\rangle=\frac{1}{\sqrt{2}}(|a\rangle|A\rangle+|b\rangle|B\rangle) \otimes|v\rangle,
$$

where $|A\rangle$ and $|B\rangle$ are states of some hypothetical third system, which may even be infinitely distant. At $t=0$ no pure state exists for the atom, yet at $t_{0}$, the state of the total system (including the field) is naturally

$$
\left|e\left(t_{0}\right)\right\rangle=\left|\psi_{0}\right\rangle \otimes \frac{1}{\sqrt{2}}\left(\left|\phi_{a}\right\rangle|A\rangle+\left|\phi_{b}\right\rangle|B\rangle\right),
$$

where $\left|\phi_{a}\right\rangle$ and $\left|\phi_{b}\right\rangle$ are the (pure) field states which are obtained at $t_{0}$ when the atom is initially prepared in $|a\rangle$ and $|b\rangle$, respectively. Equation (13) indeed shows the atom in a pure state and the field entangled with the distant system.

From these examples one can see that the JCM in the limit $\bar{n} \rightarrow \infty$ becomes an ideal device to perform a state preparation on a quantum system (the atom), regardless of this system's initial state or previous entanglement history. This is a unique example, to the best of this author's knowledge. One must mention in this context the work of Meystre and co-workers [9], who showed in a system also evolving according to the JCM dynamics how a pure state might be prepared under certain conditions. Their system is, however, the quantized electromagnetic field, whose space of states is much larger than the two-level atom's, and thus a specific state can be prepared only for initial states which satisfy some appropriate conditions. For many initial states, in fact, convergence to a pure state does not occur in the Meystre-Slosser-Braunstein system.

To conclude this section, one may remark again that even though all the initial atomic states evolve towards the pure state $\left|\psi_{0}\right\rangle$ at $t=t_{0}$, only when the initial state is one of the special states $|+\rangle$ and $|-\rangle$ does the atomic system remain in a pure state throughout its evolution. For all other states, a total or partial "collapse of the state purity" takes place at the conventional JCM "collapse time" $t_{c} \sim 1 / \mathrm{g}$ of the Rabi oscillations, as was illustrated in Ref. [12] (Fig. 1; see also Ref. [10]). One is tempted to inquire, in view of the apparent loss of memory exhibited by the atom, at $t=t_{0}$, regarding its initial state, whether a collapse of the wave function in the sense of the quantum theory of measurement takes place around $t=t_{c}$, with the information about the initial state of the atom being stored in the field. In fact, one may wonder whether in some sense a measurement of the initial state of the atom can be said to be carried out by the field at the time of collapse $t_{c}$; the field, from this perspective, would be the "apparatus" which does the measuring and stores the information. This intriguing hypothesis is thoroughly explored in the following section, where the analogies with quantum measurement theory will indeed be seen to be many and remarkable.

\section{THE JCM COLLAPSE AS A QUANTUM MEASUREMENT}

\section{A. The measurement analogy}

It has long been known $[3,4]$ that for an atom prepared initially in one of the energy eigenstates the JCM predicts Rabi oscillations of decreasing amplitude, which all but vanish around the so-called collapse time $t_{c} \sim 1 / \mathrm{g}$ [more precisely, the oscillations have a Gaussian envelope which decays as $e^{-g^{2} t^{2}}$; see below, Eq. (20)]. Partial revivals of these oscillations take place around the revival time $t_{R}=2 \pi \sqrt{\bar{n}} / g$ introduced in the preceding section. Note that for large $\bar{n}$ the collapse and revival involve completely different time scales, with $t_{c}<t_{R}$.

The short-time evolution of the JCM, where short means $t<t_{R}$, but, possibly, $t>t_{c}$, can be easily obtained from the expressions (6a) and (6b). The field states $\left|\Phi_{ \pm}(t)\right\rangle$ [see Eq. (7)] are in this limit, to a good approximation, very similar to coherent states: one has

$g t \sqrt{n}=g t \sqrt{\bar{n}}+\frac{g}{2} \frac{n-\bar{n}}{\sqrt{\bar{n}}} t-\frac{g}{8} \frac{(n-\bar{n})^{2}}{\bar{n}^{3 / 2}} t+\ldots$.

For all the number states having an appreciable weight in the sum (7), one expects $(n-\bar{n})^{2}$ to be of the order of, or smaller than, $\bar{n}$; hence, as long as $t<<t_{R}=2 \pi \sqrt{\bar{n}} / g$, the third term on the right-hand side of (14) and all the higher-order terms may be ignored to yield

$$
\begin{aligned}
\left|\Phi_{ \pm}(t)\right\rangle & =e^{-\bar{n} / 2} \sum_{n=0}^{\infty} \frac{\bar{n}^{n / 2}}{\sqrt{n !}} e^{-i n \phi} e^{\mp i g t \sqrt{n}}|n\rangle \\
& \simeq e^{\mp i g t \sqrt{\bar{n}} / 2} e^{-\bar{n} / 2} \sum_{n=0}^{\infty} \frac{\bar{n}^{n / 2}}{\sqrt{n !}} e^{-i n(\phi \pm g t / 2 \sqrt{\bar{n}})} \\
& =e^{\mp i g t \sqrt{\bar{n}} / 2}\left|v e^{\mp i g t / 2 \sqrt{\bar{n}}}\right\rangle,
\end{aligned}
$$

where, as in Eq. (4), $v=\sqrt{\bar{n}} e^{-i \phi}$, and the notation in the last line means a coherent state with the complex amplitude shown. It follows, then, from Eq. (6) that, for the time considered, the evolution of a system prepared initially in one of the states $| \pm\rangle|v\rangle$ may be written as

$$
\begin{aligned}
| \pm\rangle|v\rangle \rightarrow & \rightarrow e^{\mp i g t \sqrt{\bar{n}} / 2} \frac{1}{\sqrt{2}} \\
& \times\left(e^{\mp i g t / 2 \sqrt{\bar{n}}}|a\rangle \pm|b\rangle\right) \mid v e^{\mp i g t / 2 \sqrt{\bar{n}}\rangle} \\
\simeq & e^{\mp i g t \sqrt{\bar{n}} / 2}| \pm\rangle \mid v e^{\mp i g t / 2 \sqrt{\bar{n}}\rangle} .
\end{aligned}
$$

The phase factor neglected in going from the first line to the second line of Eq. (16) is very close to unity for times $t \ll t_{R}$. Around the collapse time $t_{c}$, the phase in question is of the order of $g t_{c} / 2 \bar{n}^{1 / 2} \sim 1 / \bar{n}^{1 / 2}$, and thus, for large average photon number, negligible. The same phase factor $e^{\mp i g t / 2 \sqrt{\bar{n}}}$ cannot, however, be neglected in the field coherent state in (16), due to the large (proportional to $\sqrt{\bar{n}}$ ) amplitude of that field, as will be explained 
presently.

Equation (16) shows that, over times of the order of the collapse time, the states $| \pm\rangle$ remain approximate eigenstates of $H_{I}$, i.e., approximately unaffected by the evolution. The field, on the other hand, while remaining approximately a coherent state, picks up a phase factor which grows with time and has opposite sign for the initial state $|+\rangle$ than for the initial state $|-\rangle$. (As mentioned earlier, this splitting of the field probability distribution into two counterrotating peaks appears to have been first pointed out by Eiselt and Risken [15]. It will be featured prominently in Sec. IV as well.)

Equation (16) also leads immediately to an understanding of what happens to a system, initially prepared in any state other than $| \pm\rangle|v\rangle$, at the time of the collapse. Consider, for instance, a system prepared in an initial state $(\gamma|+\rangle+\delta|-\rangle)|v\rangle$, where $\gamma$ and $\delta$ are arbitrary coefficients (in particular, the energy eigenstates $|a\rangle$ and $|b\rangle$ are given by $\gamma=e^{i \phi} / \sqrt{2}, \delta=e^{i \phi} / \sqrt{2}$ and $\gamma=1 / \sqrt{2}$, $\delta=-1 \sqrt{2}$, respectively). By the linearity of the problem, and according to Eq. (16), this must evolve as

$$
\begin{aligned}
(\gamma|+\rangle+\delta|-\rangle)|v\rangle \rightarrow & \gamma e^{-i g t \sqrt{\bar{n}} / 2}|+\rangle\left|v e^{-i g t / 2 \sqrt{\bar{n}}}\right\rangle \\
& +\delta e^{i g t \sqrt{\bar{n}} / 2}|-\rangle\left|v e^{i g t / 2 \sqrt{\bar{n}}}\right\rangle .
\end{aligned}
$$

This is in general no longer a pure state for the atom or the field; the corresponding reduced density operator for the atom is

$$
\rho_{\text {at }}(t)=|\gamma|^{2}|+\rangle\left\langle+\left.|+| \delta\right|^{2} \mid-\right\rangle\langle-|+\left(\gamma \delta^{*} e^{-i g t \sqrt{\bar{n}}}\left\langle v e^{i g t / 2 \sqrt{\bar{n}}} \mid v e^{-i g t / 2 \sqrt{\bar{n}}}\right\rangle|+\rangle\langle-|+\text { H.c. }\right) .
$$

This density operator becomes diagonal to a good approximation, as soon as $t$ is somewhat larger than the collapse time. To see this, note that the scalar product of two coherent states is given by the general formula [7]

$$
\left\langle v^{\prime} \mid v\right\rangle=\exp \left[-\left(\left|v^{\prime}\right|^{2}+|v|^{2}\right) / 2+v^{\prime *} v\right]
$$

which, for the scalar products appearing in Eq. (18), yields

$$
\begin{aligned}
& \left|\left\langle v e^{-i g t / 2 \sqrt{\bar{n}}} \mid v e^{i g t / 2 \sqrt{\bar{n}}}\right\rangle\right|^{2} \\
& =\exp \left(-4 \bar{n} \sin ^{2} g t / 2 \sqrt{\bar{n}}\right) \simeq e^{-g^{2} t^{2}},
\end{aligned}
$$

where the last approximation holds for the times we are considering, namely, $t<<t_{R}$. Clearly, when (18) becomes diagonal the oscillations in the population inversion disappear; i.e., the inversion "collapses" to zero.

The analogies with a measurement being carried out on the atom should by now become evident. In the limit $\bar{n} \rightarrow \infty$, and for times $t<<t_{R}$, the states $|+\rangle$ and $|-\rangle$ are approximate eigenstates of the interaction; any other initial atomic state is promptly (in a time of the order of the collapse time $t_{c}=1 / g$ ) reduced to a mixture which is diagonal in the basis $\{|+\rangle,|-\rangle\}$; the weights of the states in the mixture, $|\gamma|^{2}$ and $|\delta|^{2}$, correspond to the probabilities to find the atom in state $|+\rangle$ or state $|-\rangle$, respectively, upon such a measurement. Moreover, the information on the initial state of the atom is stored in the field: as Eq. (17) indicates, to the initial atomic state $|+\rangle$ corresponds a field state which is approximately the initial coherent state shifted in phase by an amount $\phi_{+}=g t / 2 \sqrt{\bar{n}}$, and to the initial atomic state $|=\rangle$ a coherent state shifted by the amount $\phi_{-}=-g t / 2 \sqrt{\bar{n}}$.

To all this, one may add the following observation: the collapse occurs as soon as the two field states become macroscopically distinguishable.

To support this contention, recall that the "quantum" or "shot-noise" limit to a precise phase measurement is given by

$$
\delta \phi=\phi_{1}-\phi_{2} \geq \frac{1}{\sqrt{\bar{n}}}
$$

when the field is in a coherent state [26]. The phase difference $\phi_{+}-\phi_{-}=g t / \sqrt{\bar{n}}$ becomes therefore just resolvable when $g t / \sqrt{\bar{n}} \geq 1 / \sqrt{\bar{n}}$, i.e., when $t \geq 1 / g$. It is at this point that one may claim to have a "macroscopic" record of the initial state of the atom, as quantum measurement theory demands. The phase of the field would play the role of the "pointer observable" (cf. Zurek's approach to quantum measurement theory [27]).

This approach offers an interesting interpretation of an old result of Milburn [29] regarding the JCM evolution when the field is initially prepared in a squeezed, rather than a coherent, state. The shot-noise limit just discussed may be understood in terms of the intrinsic phase uncertainty of a coherent state [30]. Quantized field states which exhibit a greater or smaller phase uncertainty are called squeezed states [31], and the connection between phase uncertainty and JCM collapse is nicely illustrated if one considers, as Milburn did, the field to be initially prepared in a "bright" squeezed state (that is, one with a large coherent amplitude). For moderate amounts of squeezing, such a state may have a coherent amplitude $\sim \sqrt{\bar{n}}$ and a phase uncertainty [32]

$$
\Delta \phi \sim \frac{1}{2 \sqrt{\bar{n}}} e^{-r},
$$

where $r$ is a squeezing parameter: $r>0$ for a phasesqueezed state, $r=0$ for a coherent state, and $r<0$ for an amplitude-squeezed state [which, as Eq. (22) shows, has therefore a larger phase uncertainty than a coherent state]. If the collapse takes place at the time when the phase difference $\phi_{+}-\phi_{-}=g t / \sqrt{\bar{n}}$ is just resolvable, then by (22) one expects the collapse time for a squeezed state to scale as

$$
t \sim \frac{1}{g} e^{-r} .
$$

Hence the collapse would take place faster (relative to a 
coherent state) for an initial phase squeezed state (whose phase is better defined), and more slowly for an amplitude squeezed state $(r<0)$, whose phase is less precisely defined. This is indeed what Milburn found [29]. (This result, of course, may also be understood, in what one might call a complementary way, from the traditional argument [3] which relates the collapse to the spread in photon number in the field state: see Milburn for the details.)

It would appear from the foregoing that the JCM may, quite fortuitously, offer a paradigm of a quantum measurement. There is a complete orthogonal set of atomic states $(|+\rangle$ and $|-\rangle)$ which are, in the limit $\bar{n} \gg 1$, essentially unaffected by the interaction over the time scale considered; nonetheless, the interaction does establish a strong correlation between each one of these states and specific states of the field which for sufficiently long times, and large numbers of photons, become macroscopically distinct. This last statement is true, even though the restriction $t \ll t_{R}$ prevents the phase difference $g t / \sqrt{\bar{n}}$ between the states $\left|v e^{i g t / 2 \sqrt{\bar{n}}}\right\rangle$ and $\left|v e^{-i g t / 2 \sqrt{\bar{n}}}\right\rangle$ from ever being larger than a radian, because even such a small phase difference is amply resolvable for a sufficiently intense field [see Eq. (21)]; in particular, the change in intensity associated with such a phase difference in a hypothetical interference experiment could easily be made observable. It is especially interesting in this problem that the "measuring apparatus," i.e., the field, is described fully quantum mechanically and yet possesses a well-defined classical limit; in particular, it is only in this classical limit $\bar{n} \rightarrow \infty$ that the reduced density operator for the atom (18) is seen to become diagonal in the basis $\{|+\rangle,|-\rangle\}$.

Pursuing this interpretation, it is natural to ask what is the observable being measured. The eigenstates of the measurement are clearly $|+\rangle$ and $|-\rangle$. Since the outcome of the measurement (the field phase) is positive for one of these states and negative (and equal in magnitude) for the other, it follows that the observable being measured is proportional to

$|+\rangle\langle+|-|-\rangle\left\langle-\left|=e^{-i \phi}\right| a\right\rangle\left\langle b\left|+e^{i \phi}\right| b\right\rangle\langle a|$,

which is the component of the atomic dipole moment in phase with the field. As pointed out in Sec. II, the eigenstates $|+\rangle$ and $|-\rangle$ are just those states of the atom in which the atomic dipole is exactly in phase with (or in opposition to) the field, which are the states in which, in the rotating-wave approximation, no exchange of energy between dipole and field takes place.

All of this leads naturally to an inevitable question: if this is, indeed, a model of a quantum measurement, what does it have to say about the most problematic issue in quantum measurement theory, namely, the collapse of the wave function (or reduction of the state vector)? Is there anything in the formalism developed so far that suggests that such a reduction has taken place?

\section{B. Reduction of the state vector}

The postulate of the reduction of the state vector applied to the present case would state that, after the mea- surement is over, the combined atom-field system is left in either one of the two possible "outcomes": atom in state $|+\rangle$, field in state $\left|v e^{-i \phi_{+}}\right\rangle$, or atom in state $|-\rangle$, field in state $\left|v e^{-i \phi_{-}}\right\rangle$. The difficulty is that the Schrödinger dynamics leads instead to the state (17) for the total system, where both alternatives appear to be simultaneously present.

It is by now well known that it is in general not legitimate to think of a system represented by a state vector such as (17), which is a coherent superposition of two distinct states, as being actually in either of the two states at any given time. There are by now many experiments on many systems from photons and electrons to neutrons and even whole atoms which show that such an interpretation is in general incorrect: it is essentially like thinking of a particle in a double-slit interference experiment as actually passing through a specific slit (and not through the other), when its state is in fact a coherent superposition of the two states "passage through slit 1" and "passage through slit $2 . "$

The possibility of experimentally disproving the notion that the system described by (17) is actually, at any given time, in one of the states $|+\rangle\left|v e^{-i \phi_{+}}\right\rangle$or $|-\rangle\left|v e^{-i \phi_{-}}\right\rangle$ hinges, therefore, on the possibility of setting up an experiment which would reveal interference between the two parts of the wave function (the two "alternate paths") $|+\rangle\left|v e^{-i \phi_{+}}\right\rangle$and $|-\rangle\left|v e^{-i \phi_{-}}\right\rangle$. That is, formally, such a refutation depends on the possibility of measuring an operator which has a nonvanishing matrix element between the states $|+\rangle\left|v e^{-i \phi_{+}}\right\rangle$and $|-\rangle\left|v e^{-i \phi_{-}}\right\rangle$. But what if such an operator cannot be found, or simply cannot be measured? If it is the possibility of observing interference between $|+\rangle\left|v e^{-i \phi_{+}}\right\rangle$and $|-\rangle\left|v e^{-i \phi_{-}}\right\rangle$that forbids one from thinking of the system as being either in $|+\rangle\left|v e^{-i \phi_{+}}\right\rangle$or in $|-\rangle\left|v e^{-i \phi_{-}}\right\rangle$, if interference becomes impossible to observe does it thereby become legitimate to believe that one (and not the other) of the possibilities $|+\rangle\left|v e^{-i \phi_{+}}\right\rangle$and $|-\rangle\left|v e^{-i \phi_{-}}\right\rangle$has in fact become actualized for the system, i.e., that the reduction of the state vector has taken place?

If indeed no interference between $|+\rangle\left|v e^{-i \phi_{+}}\right\rangle$and $|-\rangle\left|v e^{-i \phi_{-}}\right\rangle$could be observed, then it would be entirely justified, for an ensemble of identically prepared systems, to replace the pure-state description provided by (17) by the mixed-state description provided by the density operator

$$
\begin{aligned}
\rho_{\text {inc }}= & |\gamma|^{2}|+\rangle\left\langle+|\otimes| v e^{-i g t / 2 \sqrt{\bar{n}}}\right\rangle\left\langle v e^{-i g t / 2 \sqrt{\bar{n}}}\right| \\
& +|\delta|^{2}|-\rangle\left\langle-|\otimes| v e^{i g t / 2 \sqrt{\bar{n}}}\right\rangle\left\langle v e^{i g t / 2 \sqrt{\bar{n}}}\right| .
\end{aligned}
$$

The reason for going from a single-system description to an ensemble description is simply that, according to our hypothesis, for an individual system we cannot know which of the two possibilities $|+\rangle\left|v e^{-i \phi_{+}}\right\rangle$or $|-\rangle\left|v e^{-i \phi_{-}}\right\rangle$has become actualized. This is expressed by the operator $\rho_{\text {inc }}$. Formally, we have replaced the coherent superposition of states (17) by an incoherent one, 
namely, (25).

The statement that no physical observable (however one defines "physical observable," which may not be a trivial task) has any matrix elements between some two states amounts to a superselection rule. Some resolutions of the quantum measurement problem along the lines just sketched, i.e., involving superselection rules, have been proposed; the best known is probably Zurek's proposal [27] of "environment-induced superselection rules," but the earlier work of Pfeifer [28] is also of special interest as it involves two-level systems with Hamiltonians closely related to the present one.

It seems, in fact, at first sight that for the problem at hand one could prove a sort of "superselection rule" (and that without having to invoke an "environment") as follows: in the limit

$$
t \rightarrow \infty, \quad \bar{n} \rightarrow \infty, \frac{g t}{\sqrt{\bar{n}}} \rightarrow 0
$$

every field operator which involves finite powers of creation and annihilation operators has vanishing matrix elements between the states $\left|v e^{i g t / 2 v}\right\rangle$ and $\left|v e^{-i g t / 2 v}\right\rangle$, provided that $\bar{n}$ does not go to infinity faster than any power of $t$.

To show this, it is enough to consider normally ordered field operators, and, for example, the matrix element of $a^{p}$, which, according to Eq. (20), has magnitude

$$
\left|\left\langle v e^{-i g t / 2 \sqrt{\bar{n}}}\left|a^{p}\right| v e^{i g t / 2 \sqrt{\bar{n}}}\right\rangle\right|=\bar{n}^{p / 2} e^{-g^{2} t^{2} / 2},
$$

which goes to zero in the limit (26) (with the restriction on $\bar{n}$ mentioned above), for any finite power $p$.

This is a somewhat intriguing result. Clearly it leaves open a number of questions, such as whether operators involving an infinite number of powers of field operators (e.g., $e^{i n}$ ) ought perhaps to be regarded as nonmeasurable. It also hints at the possibility that the macroscopic nature of the apparatus itself, without having to resort to an external environment, might suffice to provide a superselection rule and with it a justification for the collapse of the wave-function postulate.

Yet, interesting as all this seems, it may unfortunately not be applicable to the real JCM, for the following reason: Eq. (17) is really only the leading term in a hypothetical expansion of the state of the system in powers of $1 / \bar{n}$. The terms neglected, while going to zero in the limit $\bar{n} \rightarrow \infty$, may well make finite contributions to expectation values of such field operators as, e.g., $n^{2}$, which neither the postulated density operator (25) nor, for that matter, the state (17) would account for. Unless these terms can be quantified somehow, the precise relevance of the result (26) and (27) to the question of the state reduction in the JCM will remain questionable: the possibility has to be allowed for that one could find an operator whose measurement would disclose the fact that (25) is not the true state of the system (and, more to the point, that the true state is not a mixture but a coherent super- position), even in the limit (26).

It might be worthwhile to pursue these ideas in some formally simpler models (such as, e.g., the so-called Raman-coupled model) where an exact solution in closed form (as opposed to just an asymptotic expression) is more readily obtained than in the JCM. In the end, perhaps the best assessment of the situation at this point is that this model offers some intriguing suggestions on the possible meaning of the reduction of the state-vector hypothesis for a quantum measurement, but that it is not clear at present whether one can really defend the notion that such a reduction does take place in the present system, even in the limit (26).

When the limit (26) is not taken, on the other hand, that is, for finite $\bar{n}$, or for times comparable to $\sqrt{\bar{n}} / g$, the incoherent mixture (25) may still be a very good approximation to the coherent superposition (17), but the two are most definitely not equivalent. This turns out to be dramatically illustrated by the well-known revival phenomenon, as will be shown in the following section.

\section{THE SCHRÖDINGER CAT AT HALF-REVIVAL TIME}

Beyond the collapse time scale $t_{c} \sim 1 / \mathrm{g}$ lies the revival time scale governed by the time of the first revival $t_{R}=2 \pi \sqrt{\bar{n}} / g$. Clearly, for these times neither the expression (17) nor (25) continue to be good approximations to the state of the system. One must return to the original expressions (6a) and (6b), and write, for the state at time $t$ of a system initially prepared in the state $(\gamma|+\rangle+\delta|-\rangle)|v\rangle$,

$$
\begin{aligned}
|\psi(t)\rangle= & \gamma\left(e^{-i \phi} e^{-i g t / 2 \sqrt{\bar{n}}}|a\rangle+|b\rangle\right)\left|\Phi_{+}(t)\right\rangle \\
& +\delta\left(e^{-i \phi} e^{i g t / 2 \sqrt{\bar{n}}}|a\rangle-|b\rangle\right)\left|\Phi_{-}(t)\right\rangle,
\end{aligned}
$$

with $\left|\Phi_{+}(t)\right\rangle$ and $\left|\Phi_{-}(t)\right\rangle$ given by Eq. (7). Then, the fact that at the time $t_{0}$ the two atomic states appearing in (28) become identical means that the state of the field at that time is a (coherent) linear superposition of the states $\left|\Phi_{+}\left(t_{0}\right)\right\rangle$ and $\left|\Phi_{-}\left(t_{0}\right)\right\rangle$ which, as will be shown presently, are macroscopically distinct states; i.e., the state of the field at $t=t_{0}$ is what has come to be called a Schrödinger cat.

Nonetheless, it is interesting to pursue for a while the line of thinking introduced in the preceding section, according to which, as long as the states $\left|\Phi_{+}(t)\right\rangle$ and $\left|\Phi_{-}(t)\right\rangle$ have negligible overlap, that is, as long as most operators one could think of measuring have negligible matrix elements between $\left|\Phi_{+}(t)\right\rangle$ and $\left|\Phi_{-}(t)\right\rangle$ (which, as shown in the preceding section, happens already some time after the conventional collapse time $t_{c}$ ), it may be a good approximation to represent the state of an ensemble of identically prepared systems, not by the pure state (28) but by the incoherent mixture

$$
\begin{aligned}
\rho_{\text {inc }}(t)= & |\gamma|^{2}\left(e^{-i \phi} e^{-i g t / 2 \sqrt{\bar{n}}}|a\rangle+|b\rangle\right)\left(e^{i \phi} e^{i g t / 2 \sqrt{\bar{n}}}\left\langle a|+\langle b|) \otimes \mid v e^{-i g t / 2 \sqrt{\bar{n}}}\right\rangle\left\langle v e^{-i g t / 2 \sqrt{\bar{n}}}\right|\right. \\
& +|\delta|^{2}\left(e^{-i \phi} e^{i g t / 2 \sqrt{\bar{n}}}|a\rangle-|b\rangle\right)\left(e^{i \phi} e^{-i g t / 2 \sqrt{\bar{n}}}\left\langle a|-\langle b|) \otimes \mid v e^{i g t / 2 \sqrt{\bar{n}}}\right\rangle\left\langle v e^{i g t / 2 \sqrt{\bar{n}}}\right|\right.
\end{aligned}
$$


The density operator (29) [a time-evolved version of (25)] lends itself to the interpretation that the original ensemble of identically prepared systems has broken up into two, in each of which the individual systems are evolving in time just as if their initial states had been $|+\rangle|v\rangle$ and $|-\rangle|v\rangle$, respectively. If, indeed, (29) can be shown to be approximately correct under appropriate circumstances, this is an interesting result, because it means that, far from being special cases of limited interest, the product-state trajectories $(6 \mathrm{a})$ and $(6 \mathrm{~b})$ can be taken as generic, in the sense that, as long as the approximation (29) holds, it is legitimate to think of an ensemble of systems, regardless of in which state they are originally prepared, as being reduced, after the collapse time, to two subensembles each of which evolves along one of the trajectories (6a) and (6b). A study of these trajectories, therefore, holds the key to many of the properties of the JCM, regardless of the initial state of that atom, after the collapse time. [For example, it is obvious that as long as (29) holds the population inversion is zero, since both atomic states appearing in (29) correspond to equal population of the levels $|a\rangle$ and $|b\rangle$.]

To assess the goodness of (29) as well as its limitations, it is necessary to look at the field states $\left|\Phi_{+}(t)\right\rangle$ and $\left|\Phi_{-}(t)\right\rangle$ in more detail. The approximate result (15), which identifies them with coherent states with counterrotating phases, holds only for times short compared to the revival time $t_{R}$ and hence short compared to the half-revival time $t_{0}=t_{R} / 2$. The reason is that for these long times, of the order of $\sqrt{\bar{n}} / g$, the quadratic term in the expansion of the square root in Eq. (14) can no longer be neglected, since $(n-\bar{n})^{2}$ can be of the order of $\bar{n}$ for the Poisson distribution, which makes $g t_{0}(n-\bar{n})^{2} / 8 \bar{n}^{3 / 2} \sim 1$.

The higher-order terms may still be neglected, but the quadratic term is enough to make the states $\left|\Phi_{+}(t)\right\rangle$ and $\left|\Phi_{-}(t)\right\rangle$ look rather different from coherent states for large $t$. It is clear, however, that they have a number of properties in common with coherent states. (In fact, as mentioned in Appendix B, they are members of a class of states which have been called "generalized coherent states" and which have been the subject of some, rather general, theoretical studies [33].) For one thing, from the definition (7) they are immediately seen to have exactly the same photon-number distribution as the initial coherent state $|v\rangle$ (note, however, that this is not at all true for their linear superpositions). The expectation values of field operators in the states $\left|\Phi_{ \pm}(t)\right\rangle$ are also to leading order in $\bar{n}$ what one would expect from the coherent states (15). (See Appendix B, where these and other properties of these states, including squeezing, are studied in greater detail.) One way to describe them which illustrates quite clearly the main differences with coherent states, and also makes contact with the work of previous authors, is by plotting the quasiprobability distribution called the $Q$ function,

$$
Q_{ \pm}(\alpha, t)=\left|\left\langle\alpha \mid \Phi_{ \pm}(t)\right\rangle\right|^{2} .
$$

Contour plots of this function for different times are shown in Fig. 1, and they may be compared with those

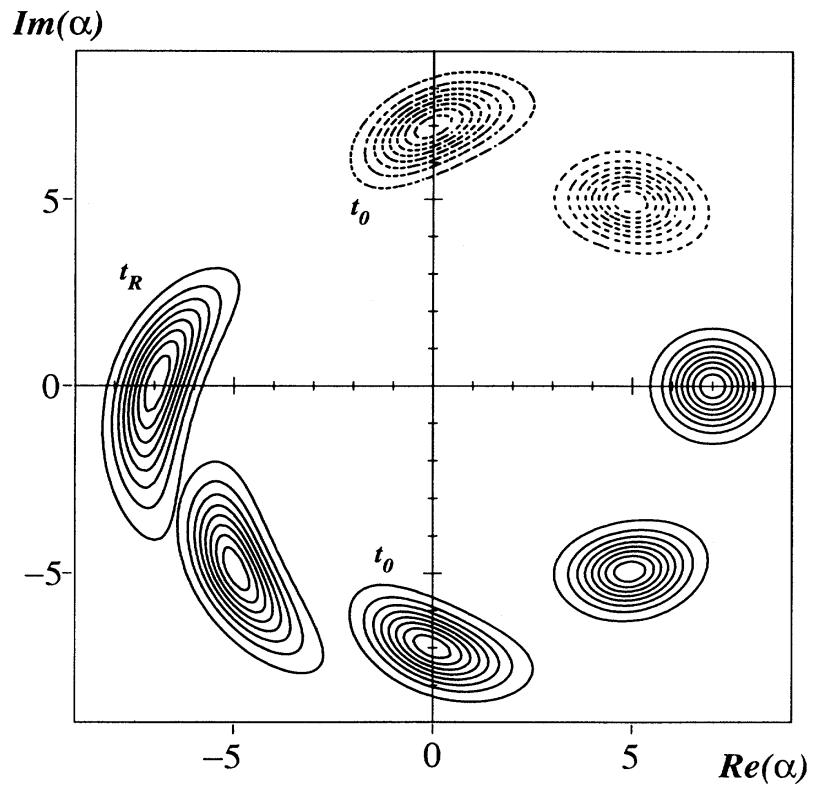

FIG. 1. Contour plots of the $Q$ function for the states $\left|\Phi_{+}(t)\right\rangle$ (solid lines) and $\left|\Phi_{-}(t)\right\rangle$ (dashed lines). The first, which moves clockwise, is shown for the times $t=0, t_{R} / 4$, $t_{0} \equiv t_{R} / 2,3 t_{R} / 4$, and $t_{R}$; the second (counterclockwise) is plotted only for the times $t=0$ (where the two coincide), $t_{R} / 4$, and $t_{0} \equiv t_{R} / 2$. The average photon number is 49 .

shown by Eiselt and Risken [16]. These authors predicted the basic behavior, with $Q_{+}$rotating clockwise and $Q_{-}$counterclockwise, in maximum opposition (fields $180^{\circ}$ out of phase) at half-revival time $t_{0}$ and overlapping again at the revival time $t_{R}$. This is also consistent with the study of phase properties in the field in the JCM in Ref. [17]; see also Ref. [18] for the Raman-coupled model. It does not appear to have been noticed, however, in the previous studies that in the JCM in the limit $\bar{n} \rightarrow \infty$ these plots describe in fact pure field states, i.e., that along either the clockwise or the counterclockwise branch the $Q$ function, which is generally given by $Q \equiv\left\langle\alpha\left|\rho_{\text {field }}\right| \alpha\right\rangle$, is of the special pure-state form $\left|\left\langle\alpha \mid \Phi_{ \pm}(t)\right\rangle\right|^{2}$ given in Eq. (30), with $\left|\Phi_{ \pm}(t)\right\rangle$ being the states defined in Eq. (6).

Most interesting of all is perhaps what happens at the half-revival time in this limit: if the initial atomic state is a linear superposition of $|+\rangle$ and $|-\rangle$, the $Q$ function for the field at time $t_{0}$ will exhibit contours widely apart in the phase plane, like the top and bottom ones (marked $t_{0}$ ) in Fig. 1. Yet this superposition of widely different field states, $\left|\Phi_{+}(t)\right\rangle$ and $\left|\Phi_{-}(t)\right\rangle$, is in fact a pure state, because of the identity of the atomic states in the superposition (28) at $t=t_{0}$.

Suppose, for definiteness, that the initial state of the atom is the upper energy state $|a\rangle$ $=e^{i \phi}(|+\rangle+|-\rangle) / \sqrt{2}$; then at $t \sim t_{0}$ we have

$\left|\psi\left(t_{0}\right)\right\rangle=e^{i \phi}\left|\psi_{0}\right\rangle \otimes \frac{1}{\sqrt{2}}\left[\left|\Phi_{+}\left(t_{0}\right)\right\rangle-\left|\Phi_{-}\left(t_{0}\right)\right\rangle\right]$ 
[with the atomic state $\left|\psi_{0}\right\rangle$ given by Eq. (9)], that is, a pure field state which is a linear superposition of two macroscopically distinct states (described by the top and bottom quasiprobability distributions in the phasor space of Fig. 1). As mentioned above, such states have been dubbed Schrödinger cats. (It may be worth mentioning, however, that the state (17), discussed at some length in Sec. III, is in fact much closer to the spirit of Schrödinger's original [34] cat, being an entangled state in which two alternative states of a microscopic system (the atom) are correlated with two macroscopically distinct states of a macroscopic system (the field, which therefore plays the role of the cat).)

It is interesting to notice the similarity between the present "cat" and the one proposed by Yurke and Stoler [19], which also may be viewed as a superposition of two states of opposite phase. There is in fact a formal analogy between the states $\left|\Phi_{ \pm}(t)\right\rangle$, when the quadratic approximation for $\sqrt{n}$ in the exponent is used, and the states resulting from the evolution of an anharmonic oscillator [35] or a field interacting with a Kerr medium [14]; see Appendix B for further details.

There have been other proposals to produce Schrödinger cat field states, in the sense of (31), which also rely on JCM dynamics: see the papers quoted in Ref. [9]. The present results indicate that a single atom, initially in the ground or excited state, interacting with a micromaser-cavity field in an initially coherent state, produces by itself a Schrödinger-cat-like state after an interaction time $t_{0}=t_{R} / 2$. Experimentally, the main difficulty may be in producing an initial coherent-statelike field in the cavity; a good possibility might be to use an appropriate cotangent state [9], which can be very close to a coherent state for the right choice of parameters. Of course, it might be argued that if one were able to prepare a single-peaked cotangent state in the cavity, one might probably just as easily prepare a doublypeaked, Schrödinger-cat-like one [9].

One of the questions that is often asked about such superpositions of macroscopically distinct states is how they could be observed; that is, given the very small (in the macroscopic limit, infinitesimal) overlap between the states $\left|\Phi_{+}\left(t_{0}\right)\right\rangle$ and $\left|\Phi_{-}\left(t_{0}\right)\right\rangle$, what kind of experiment could reveal that the state $(31)$ is really a coherent superposition, rather than an incoherent mixture such as

$$
\begin{aligned}
\rho_{\text {inc }}\left(t_{0}\right)=\left|\psi_{0}\right\rangle\left\langle\psi_{0}\right| \otimes \frac{1}{2}[ & \left|\Phi_{+}\left(t_{0}\right)\right\rangle\left\langle\Phi_{+}\left(t_{0}\right)\right| \\
+ & \left.\left|\Phi_{-}\left(t_{0}\right)\right\rangle\left\langle\Phi_{-}\left(t_{0}\right)\right|\right] .
\end{aligned}
$$

This is, of course, just a special case of the question of to what extent is the mixed state (29) a good approximation to the "real" pure state (28), in general, and in fact whether it is so good as to be, for practical purposes, indistinguishable. It is also the same question (coherent superpositions versus incoherent mixtures) which was asked in the context of the reduction of the state vector hypothesis in Sec. II. There it was asserted that in a certain limit, given by (26), the two descriptions were for all practical purposes indistinguishable. It is interesting to notice that, in effect, what the limit (26) does is to push the revival time infinitely into the future, so as to make it inaccessible.

If that limit is not taken, a very simple way to observe the difference between the coherent linear superposition (31) and the incoherent mixture (32) is, in fact, to wait until the revival time $t_{R}$. At that time, always according to the evolution equations (6), the state (31) has evolved into

$\left|\psi\left(t_{R}\right)\right\rangle=-e^{i \phi} \frac{1}{\sqrt{2}}\left[|-\rangle\left|\Phi_{+}\left(t_{R}\right)\right\rangle+|+\rangle\left|\Phi_{-}\left(t_{R}\right)\right\rangle\right]$

[note how, according to (6), at $t=t_{R}=2 \pi \sqrt{\bar{n}} / g$, the initial atomic state $|+\rangle$ has turned into $|-\rangle$, and vice versa], whereas the density operator (32) becomes

$$
\begin{aligned}
\rho_{\text {inc }}\left(t_{R}\right)=\frac{1}{2}[ & -\rangle\left\langle-|\otimes| \Phi_{+}\left(t_{R}\right)\right\rangle\left\langle\Phi_{+}\left(t_{R}\right)\right| \\
& \left.+|+\rangle\left\langle+|\otimes| \Phi_{-}\left(t_{R}\right)\right\rangle\left\langle\Phi_{-}\left(t_{R}\right)\right|\right] .
\end{aligned}
$$

The crucial, observable difference between these two states is that an ensemble described by (33) exhibits oscillations of the population inversion (the well-known JCM revival) whereas one described by (34) does not. This follows immediately from a consideration of the population difference operator

$$
|a\rangle\langle a|-| b\rangle\langle b|=|+\rangle\langle-|+|-\rangle\langle+| \text {. }
$$

The operator (35) always has zero expectation value in the state (34), while its expectation value in the state (33) is proportional to the scalar product

$$
\left\langle\Phi_{+}\left(t_{R}\right) \mid \Phi_{-}\left(t_{R}\right)\right\rangle \text {, }
$$

which is not at all zero, and in fact is quite significant. An extrapolation of the upper part of Fig. 1 (dashed curves) to the revival time $t_{R}$ already suggests the large overlap between the states $\left|\Phi_{+}\left(t_{R}\right)\right\rangle$ and $\left|\Phi_{-}\left(t_{R}\right)\right\rangle$ at this time. If the approximation (15) is used, in fact, one obtains easily $\left\langle\Phi_{+}\left(t_{R}\right) \mid \Phi_{-}\left(t_{R}\right)\right\rangle=1$, which would imply perfect revivals; in reality, the quadratic terms neglected in the exponent in (15) bring the value of $\left\langle\Phi_{+}\left(t_{R}\right) \mid \Phi_{-}\left(t_{R}\right)\right\rangle$ down to about 0.55 , and the revival, though substantial, is not quite perfect $[3,4]$.

The implication of this result is that the replacement of (31) by (32), which might appear to be totally justified at $t_{0}$ in view of the small overlap between the states $\left|\Phi_{+}\left(t_{0}\right)\right\rangle$ and $\left|\Phi_{-}\left(t_{0}\right)\right\rangle$, leads to a quite wrong prediction for the behavior of the system at time $t_{R}$; in fact, to a total absence of revivals. The very small overlap between the states $\left|\Phi_{+}\left(t_{0}\right)\right\rangle$ and $\left|\Phi_{-}\left(t_{0}\right)\right\rangle$ (two macroscopically distinct states of the field, as far apart as they can possibly be in phase) is thus essential to the revival phenomenon. The mutual coherence between the two pieces of the wave function (31), which appears all but lost at the time $t=t_{0}$, is in fact the basis for the population inversion revivals. Or, to put it another way: the revival phenomenon is an indirect observation of the macroscopic coherent superposition (Schrödinger cat) which was the state of the system at the earlier time $t=t_{0}$.

Various authors have dealt with the question of how the cavity losses affect the JCM revivals [36,20]. The conclusion is that even a small amount of losses suffices to wipe out the revivals completely. In view of the foregoing, it is not hard to understand why: the revival is en- 
tirely due to the very small coherence between the macroscopically distinct states $\left|\Phi_{+}\left(t_{0}\right)\right\rangle$ and $\left|\Phi_{-}\left(t_{0}\right)\right\rangle$, and it is well known [37] how easily such macroscopic superpositions are destroyed (i.e., made incoherent) by the interaction with the environment. (See also Ref. [19] and references therein.)

Indeed, some authors have seen in this fact a possible resolution to the difficulties inherent in the postulate of the collapse of the wave function: according to this viewpoint (see, e.g., Ref. [27]) it is the interaction with the environment that renders essentially inobservable the coherence between the two macroscopically distinct parts of the wave function, and thus ensures the replacement of a state vector such as (31) by a mixed-case density operator such as (32) is justified for all practical purposes. Returning for a moment to the discussion in Sec. III on the collapse of the wave function, it might be argued, along these lines, that it is only after the cavity losses make the revivals unobservable that one may, for all practical purposes, regard the collapse of the state vector and hence the measurement, as having "really taken place": the evolution of the system would then be given by an appropriately modified (to include the effect of the losses) version of the mixed ensembles (25) or (at a later time) (29). Without losses one would have a sort of "incomplete measurement," or an "incomplete collapse of the wave function" at the collapse time, which is undone at the revival time.

This argument may be combined with the macroscopic, "thermodynamic" limit of Sec. III, in the following way: in the limit in which $\bar{n} \rightarrow \infty$, the revivals are pushed infinitely far in the future. To observe them, that is, to have evidence for the coherence of the two macroscopically distinct parts of the wave function, one must ensure that the system remains isolated for an increasingly long time; this, of course, becomes impossible as $t_{R} \rightarrow \infty$.

The above description, however, may not be entirely applicable to the JCM, because, as was pointed out in Sec. III, it cannot be entirely ruled out that one might perhaps be able to tell that the state of the system is not the mixed ensemble (25) or (29) by some other, conceivable measurements [rather than having to wait (for an infinite time) for the revival] because of the asymptotic nature of the results (6a) and (6b), on which all these considerations ultimately rest. It seems that these points, i.e., the relative importance of the roles played by the macroscopic limit and the coupling to the environment, might be clarified further by looking at some other models, perhaps physically less realistic but with simpler algebra than the JCM, where the problem of the influence of the neglected terms in the asymptotic form (6), mentioned in Sec. III, might not arise. It is also a question for the future to determine in which way the use of the asymptotic states $\left|\Phi_{+}(t)\right\rangle$ and $\left|\Phi_{-}(t)\right\rangle$ may or may not simplify a calculation of the influence of losses in the JCM.

\section{CONCLUSIONS AND OUTLOOK}

The results presented in this paper may have some relevance to some areas beyond the usual scope of quantum optics and the JCM. This section briefly summarizes some possibilities for further research.

The result that for an interacting quantum system an approximately pure state description may hold in spite of the interaction, and that the resulting trajectory is not constrained to satisfy the expectations arising from the usual unitary evolution of closed systems (in particular, such things as the crossing of trajectories become possible) may have some relevance to studies of quantum dynamics in general, especially for open (dissipative) systems, and perhaps even for chaos. It is significant that these trajectories are "generic" in the sense indicated at the beginning of Sec. IV, namely, in that an ensemble of systems initially prepared in any state will, after some initial evolution (in this case, after the collapse time), be approximately well described by a mixture of subensembles of systems evolving precisely along these pure-state trajectories. In other words, every system may, in this approximation, be regarded as evolving along either (6a) or (6b) after the collapse time, regardless of what state it was initially prepared in. (The limitations of such a viewpoint have been explored in Sec. IV; in particular, as pointed out there, this approximation entirely misses the revival of the population inversion oscillations. Nonetheless, for times between the collapse and revival time it may be a very good approximation indeed.)

It seems, therefore, that some progress could be made, in general, in the study of the dynamics of a quantum system coupled to a large, "quasiclassical" system by searching for approximate solutions of the product form such as those found here for the JCM. These correspond to initial states which retain their purity as they evolve and which may therefore be called "long-lived" states of the system; they may change with time but in a predictable manner, not becoming a random ensemble after a short time unlike most other initial states or doing so only on a much longer timescale.

From the point of view of the quantum theory of measurement, the JCM has been shown to be "almost" a paradigm: there is, at the collapse time, a strong correlation between the initial state of the atom and macroscopically distinct states of a "pointer" system or apparatus, namely, the quantized electromagnetic field; and a sort of superselection rule holding in the limit of an infinitely large apparatus $(\bar{n} \rightarrow \infty)$ could almost be proven. The world "almost" here refers to the fact that these results could only be established for the asymptotic solutions, Eqs. (6), and there is some uncertainty as to how they might be modified by the inclusion of the terms neglected (which go to zero as $\bar{n} \rightarrow \infty$, but might make contributions to the expectation values of operators involving high powers of $n$, the photon number).

It seems that it might be worthwhile to investigate possible measurement analogies and state reduction in other similar, related systems where an exact closed-form solution, not depending on an asymptotic limit, were available. Some of the questions suggested by the JCM when seen from the point of view of quantum measurement theory are intriguing: in particular, one may mention the connection between the size of the apparatus and the superselection rule, and the fact that it would seem to be possible to obtain such a rule without invoking the in- 
teraction with the environment; the fact that the limit $\bar{n} \rightarrow \infty$, which is essentially a thermodynamic limit where the number of particles (photons) becomes infinite, has the effect of pushing the quantum revivals (recurrences?) infinitely far into the future; the possibility of imperfect or incomplete measurements for finite $\bar{n}$, with the attending Schrödinger cats; the revival as a manifestation of the fact that the collapse of the wave function is not altogether complete; and no doubt many others, such as the action of the environment when cavity losses are included. It seems especially interesting that here one has a measuring apparatus which may be described fully quantum mechanically, for which a classical limit exists, but for which the opposite limit (small $\bar{n}$ ) can also be envisioned.

Finally, there may be interesting applications of the results presented here in pure quantum optical research. It would be interesting, for instance, to see whether use of the special solutions (6) may simplify the calculations when the cavity losses are included in the model. The extension of these results to the case of finite atom-field detuning is in preparation. The search for similar solutions for the many theoretical variations on the JCM (e.g., Raman-coupled [38], two-photon [39], Buck-Sukumar model [40]) might yield interesting results. Also, though not explicitly shown here, it is possible to extend the basic asymptotic results to the case where the initial field is a highly excited, moderately squeezed state. Finally, there is also, from a more formal point of view, room for further research into the properties of the generalized coherent states $\left|\Phi_{+}(t)\right\rangle$ and $\left|\Phi_{-}(t)\right\rangle$.

\section{ACKNOWLEDGMENTS}

I have benefited from many conversations and useful discussions with my colleagues at the University of Arkansas, including Min Xiao, M. Lieber, and W. G. Har- ter. Thanks are also due to S. J. D. Phoenix and P. L. Knight, for bringing to my attention their Ref. [10], whose considerable relevance to the research reported here and in Ref. [12] had originally escaped my notice. This research has been supported by the Arkansas Science and Technology Authority.

\section{APPENDIX A: DERIVATION OF THE ASYMPTOTIC SOLUTION}

The exact solution for the state vector of the atom-field system in the Jaynes-Cummings model is [Eq. (1)]

$$
\begin{aligned}
|\psi(t)\rangle=\sum_{n=0}^{\infty}\{[ & \alpha C_{n} \cos (g \sqrt{n+1} t) \\
& \left.\quad-i \beta C_{n+1} \sin (g \sqrt{n+1} t)\right]|a\rangle \\
+ & {\left[-i \alpha C_{n-1} \sin (g \sqrt{n} t)\right.} \\
& \left.\left.+\beta C_{n} \cos (g \sqrt{n} t)\right]|b\rangle\right\}|n\rangle,
\end{aligned}
$$

where the initial state of the atom is $|\psi(0)\rangle_{\text {atom }}=\alpha|a\rangle+\beta|b\rangle$ and the initial state of the field is $|\psi(0)\rangle_{\text {field }}=\sum_{n=0}^{\infty} C_{n}|n\rangle$. The purpose of this appendix is to derive some approximate expressions for the state (A1) which hold in the limit in which $\bar{n}$, the average number of photons in the field, approaches infinity.

When the initial state of the field is a coherent state

$$
|\psi(0)\rangle_{\text {field }}=|v\rangle=e^{-|v|^{2} / 2} \sum_{n=0}^{\infty} \frac{v^{n}}{\sqrt{n !}}|n\rangle
$$

one has

$$
C_{n}=e^{i \phi}\left(\frac{n+1}{\bar{n}}\right)^{1 / 2} C_{n+1}
$$

where $v=\sqrt{\bar{n}} e^{-i \phi}$. One can therefore write

$$
\begin{aligned}
|\psi(t)\rangle=\sum_{n=0}^{\infty}\{ & C_{n+1}\left[\alpha e^{i \phi}\left[\frac{n+1}{\bar{n}}\right]^{1 / 2} \cos (g \sqrt{n+1} t)-i \beta \sin (g \sqrt{n+1} t)\right]|a\rangle \\
& \left.+C_{n}\left[-i \alpha e^{i \phi}\left[\frac{n}{\bar{n}}\right]^{1 / 2} \sin (g \sqrt{n} t)+\beta \cos (g \sqrt{n} t)\right]|b\rangle\right\}|n\rangle .
\end{aligned}
$$

It seems intuitively obvious that in the limit as $\bar{n} \rightarrow \infty$ one should be able to replace the factors of $(n / \bar{n})^{1 / 2}$ by unity, since the spread $\Delta n$ of the Poisson distribution about the mean $\bar{n}$ is only of the order of $\sqrt{\bar{n}}$. In fact, it is not hard to prove that the difference between the vector (A4) and the vector

$$
\left|\psi^{\prime}(t)\right\rangle=\sum_{n=0}^{\infty}\left\{C_{n+1}\left[\alpha e^{i \phi} \cos (g \sqrt{n+1} t)-i \beta \sin (g \sqrt{n+1} t)\right]|a\rangle+C_{n}\left[-i \alpha e^{i \phi} \sin (g \sqrt{n} t)+\beta \cos (g \sqrt{n} t)\right]|b\rangle\right\}|n\rangle,
$$

has vanishing norm in the limit $\bar{n} \rightarrow \infty$. Indeed, the difference

$$
\begin{aligned}
|\delta \psi\rangle \equiv|\psi(t)\rangle-\left|\psi^{\prime}(t)\right\rangle= & \alpha e^{i \phi} \sum_{n=0}^{\infty} C_{n+1}\left[\left[\frac{n+1}{\bar{n}}\right]^{1 / 2}-1\right] \cos (g \sqrt{n+1} t)|a\rangle|n\rangle \\
& +\alpha e^{i \phi} \sum_{n=0}^{\infty} C_{n}\left[\left[\frac{n}{\bar{n}}\right]^{1 / 2}-1\right] \sin (g \sqrt{n} t)|b\rangle|n\rangle
\end{aligned}
$$


and each one of the sums on the right-hand side of Eq. (A6) defines a vector of vanishing norm in the limit $\bar{n} \rightarrow \infty$. Consider the first sum, for instance,

$$
\begin{aligned}
&\left.\mid \alpha e^{i \phi} \sum_{n=0}^{\infty} C_{n+1}\left[\mid \frac{n+1}{\bar{n}}\right]^{1 / 2}-1\right]\left.\cos (g \sqrt{n+1} t)|a\rangle|n\rangle\right|^{2} \\
&=|\alpha|^{2} \sum\left|C_{n}\right|^{2} \frac{(\sqrt{n}-\sqrt{\bar{n}})^{2}}{\bar{n}} \cos ^{2}(g \sqrt{n} t)\left\langle|\alpha|^{2} \sum\left|C_{n}\right|^{2} \frac{(\sqrt{n}-\sqrt{\bar{n}})^{2}}{\bar{n}}\right. \\
&=|\alpha|^{2} \sum \frac{\left|C_{n}\right|^{2}}{\bar{n}}\left|\frac{n-\bar{n}}{\sqrt{n}+\sqrt{\bar{n}}}\right|^{2} \leq|\alpha|^{2} \frac{1}{\bar{n}^{2}}\left\langle(n-\bar{n})^{2}\right\rangle
\end{aligned}
$$

where the last inequality follows from $\sqrt{n+1}+\sqrt{\bar{n}} \geq \sqrt{\bar{n}}$ for all $n$. Clearly the right-hand side of (A7) goes to zero as $\bar{n} \rightarrow \infty$, since $\left\langle(n-\bar{n})^{2}\right\rangle=\bar{n}$. The same proof applies to the second sum on the right-hand side of (A6).

In the limit $\bar{n} \rightarrow \infty$, therefore, one may replace the exact solution (A1) by the approximation (A5). Consider then the evolution of the initial atomic state $|+\rangle$ defined by

$$
|+\rangle=\frac{1}{\sqrt{2}}\left(e^{-i \phi}|a\rangle+|b\rangle\right) \text {. }
$$

Substitution of $\alpha=e^{-i \phi} / \sqrt{2}$ and $\beta=1 / \sqrt{2}$ in (A5) yields

$$
\begin{aligned}
\left|\psi^{\prime}(t)\right\rangle & =\frac{1}{\sqrt{2}} \sum_{n=0}^{\infty}\left(C_{n+1} e^{-i g \sqrt{n+1} t}|a\rangle+C_{n} e^{-i g t \sqrt{n}}|b\rangle\right)|n\rangle \\
& =\frac{1}{\sqrt{2}} \sum_{n=0}^{\infty} C_{n} e^{-i g t \sqrt{n}}\left[e^{-i \phi}\left[\frac{\bar{n}}{n+1}\right]^{1 / 2} e^{-i g(\sqrt{n+1}-\sqrt{n}) t}|a\rangle+|b\rangle\right]|n\rangle .
\end{aligned}
$$

In the limit $\bar{n} \rightarrow \infty$, the term in square brackets on the second line of Eq. (A9) becomes to a good approximation independent of $n$. This may be seen as follows. First, note that the term $[\bar{n} /(n+1)]^{1 / 2}$ may be replaced by unity along the same lines as with the vector (A4). We have

$$
\begin{aligned}
\left.\mid-\frac{1}{\sqrt{2}} \sum_{n=0}^{\infty} C_{n} e^{-i g t \sqrt{n}} e^{-i \phi}\left[\mid \frac{\bar{n}}{n+1}\right]^{1 / 2}-1\right]\left.e^{-i g(\sqrt{n+1}-\sqrt{n}) t|a\rangle|n\rangle}\right|^{2} \\
=\frac{1}{2} \sum\left|C_{n}\right|^{2} \frac{(\sqrt{n+1}-\sqrt{\bar{n}})^{2}}{n+1}=\frac{1}{2} \sum\left|C_{n+1}\right|^{2} \frac{(\sqrt{n+1}-\sqrt{\bar{n}})^{2}}{\bar{n}},
\end{aligned}
$$

which clearly is bound by $1 / 2 \bar{n}$, just as Eq. (A7) was. Therefore, in the limit $\bar{n} \rightarrow \infty$, the difference between the vector $\left|\psi^{\prime}(t)\right\rangle$ [Eq. (A9)] and the vector

$$
\left|\psi^{\prime \prime}(t)\right\rangle=\frac{1}{\sqrt{2}} \sum_{n=0}^{\infty} C_{n} e^{-i g t \sqrt{n}}\left(e^{-i \phi} e^{-i g(\sqrt{n+1}-\sqrt{n}) t}|a\rangle+|b\rangle\right)|n\rangle
$$

becomes negligible.

Next, consider the difference $\sqrt{n+1}-\sqrt{n}$ in the exponential in (A11). One can show that this is approximately independent of $n$ by using the equality

$$
\begin{aligned}
\sqrt{n+1} & =\sqrt{n}+\frac{1}{\sqrt{\bar{n}}}\left(\frac{\sqrt{\bar{n}}}{\sqrt{n+1}+\sqrt{n}}\right) \\
& =\sqrt{n}+\frac{1}{2 \sqrt{\bar{n}}}+\frac{1}{2 \sqrt{\bar{n}}}\left(\frac{2 \sqrt{\bar{n}}-\sqrt{n}-\sqrt{n+1}}{\sqrt{n+1}+\sqrt{n}}\right) \\
& =\sqrt{n}+\frac{1}{2 \sqrt{\bar{n}}}+\frac{1}{2 \sqrt{\bar{n}}} \frac{1}{\sqrt{n+1}+\sqrt{n}}\left(\frac{\bar{n}-n}{\sqrt{\bar{n}}+\sqrt{n}}+\frac{\bar{n}-n-1}{\sqrt{\bar{n}}+\sqrt{n+1}}\right) .
\end{aligned}
$$

The idea is to show that the third term in Eq. (A12) becomes negligible as $\bar{n} \rightarrow \infty$, under certain restrictions on $t$. The complicated appearance of Eq. (A12) is due to a desire to avoid direct use of a Taylor-series expansion, so as not to have to worry about the magnitude of any neglected higher-order terms.

Introducing the two angles 


$$
\begin{aligned}
& \phi_{1}=\frac{g t}{2 \sqrt{\bar{n}}} \frac{\bar{n}-n}{\sqrt{n+1}+\sqrt{n}}\left(\frac{1}{\sqrt{\bar{n}}+\sqrt{n}}+\frac{1}{\sqrt{\bar{n}}+\sqrt{n+1}}\right), \\
& \phi_{2}=-\frac{g t}{2 \sqrt{\bar{n}}} \frac{1}{(\sqrt{n+1+\sqrt{n}})} \frac{1}{(\sqrt{\bar{n}}+\sqrt{n+1})},
\end{aligned}
$$

one can write Eq. (A11) as

$$
\left|\psi^{\prime \prime}(t)\right\rangle=\frac{1}{\sqrt{2}} \sum_{n=0}^{\infty} C_{n} e^{-i g t \sqrt{n}}\left\{e^{-i \phi} e^{-i g t / 2 \sqrt{\bar{n}}}\left[\left(e^{i \phi_{1}} e^{i \phi_{2}}-e^{i \phi_{1}}\right)+\left(e^{i \phi_{1}}-1\right)+1\right]|a\rangle+|b\rangle\right\}|n\rangle,
$$

where the terms in parentheses inside the square brackets ( $e^{i \phi_{1}}$ and 1 have been added and subtracted) define two vectors whose norm goes to zero as $\bar{n} \rightarrow \infty$. This can be shown easily for the first one:

$$
\begin{aligned}
&\left.\left|\frac{1}{\sqrt{2}} \sum_{n=0}^{\infty} C_{n} e^{-i g t \sqrt{n}} e^{-i \phi} e^{-i g t / 2 \sqrt{\bar{n}}}\left(e^{i \phi_{1}} e^{i \phi_{2}}-e^{i \phi_{1}}\right)\right| a\right\rangle\left.|n\rangle\right|^{2} \\
&=2 \sum_{n=0}^{\infty}\left|C_{n}\right|^{2} \sin ^{2}\left(\phi_{2} / 2\right) \leq \frac{1}{2} \sum\left|C_{n}\right|^{2} \phi_{2}^{2}=\frac{g^{2} t^{2}}{8 \bar{n}} \sum\left|C_{n}\right|^{2} \frac{1}{(\sqrt{n+1}+\sqrt{n})^{2}} \frac{1}{(\sqrt{\bar{n}}+\sqrt{n+1})^{2}} \\
& \leq \frac{g^{2} t^{2}}{8 \bar{n}} \sum\left|C_{n}\right|^{2} \frac{1}{\bar{n}}=\frac{g^{2} t^{2}}{8 \bar{n}^{2}} .
\end{aligned}
$$

The right-hand side of Eq. (A15) goes to zero when $\bar{n} \rightarrow \infty$, for any $t$; one may contemplate the limit $t \rightarrow \infty$ provided that $t$ go to infinity more slowly than $\bar{n}$.

The proof that the second term in (A14) also has vanishing norm is somewhat more involved. One has

$$
\left.\left|\frac{1}{\sqrt{2}} \sum_{n=0}^{\infty} C_{n} e^{-i g t \sqrt{n}} e^{-i \phi} e^{-i g t / 2 \sqrt{\bar{n}}}\left(e^{i \phi_{1}}-1\right)\right| a\right\rangle\left.|n\rangle\right|^{2}=2 \sum_{n=0}^{\infty}\left|C_{n}\right|^{2} \sin ^{2}\left(\phi_{1} / 2\right) \text {. }
$$

An upper bound for the right-hand side of Eq. (A16) may be obtained as follows. The summation range is broken up at $n_{0}$, some suitable value of $n$ to be specified later. From $n=0$ to $n_{0}$, the upper bound chosen for $\sin ^{2}\left(\phi_{1} / 2\right)$ can be just unity, whereas from $n=n_{0}$ to infinity the upper bound $\sin ^{2}\left(\phi_{1} / 2\right) \leq\left(\phi_{1}\right)^{2} / 4$ is used. The value of $n_{0}$ is chosen so that the total probability $\Sigma_{n}\left|C_{n}\right|^{2}$ between $n=0$ and $n_{0}$ be sufficiently small. The following result (a variation of Tchebycheff's inequality; see Ref. [41]) applies in general: if $x$ is a random variable with mean $m$, standard deviation $\sigma$, and fourth moment about the mean $m_{4}$, the probability to find $x$ a distance $r \sigma$ away from the mean (where $r$ is any positive number) is

$$
\operatorname{Pr}\{|x-m|>r \sigma\} \leq \frac{m_{4}-\sigma^{2}}{m_{4}+r^{4} \sigma^{4}-2 r^{2} \sigma^{4}} .
$$

For the Poisson distribution, with mean $m=\bar{n}$, one has $m_{4} \equiv\left\langle(n-\bar{n})^{4}\right\rangle=\bar{n}+3 \bar{n}^{2}$. Using this, together with $\sigma^{2}=\bar{n}$, it is easy to see that to make the right-hand side of Eq. (A 17) smaller than $1 / \bar{n}$, it suffices to have

$$
r>(1+\sqrt{2 \bar{n}-1-1 / \bar{n}})^{1 / 2} \text {. }
$$

Then choose

$$
n_{0}=\bar{n}-\sqrt{\bar{n}}(1+\sqrt{2 \bar{n}})^{1 / 2}
$$

to have

$$
\sum_{n=0}^{n_{0}}\left|C_{n}\right|^{2}<\operatorname{Pr}\left\{|n-\bar{n}|>\bar{n}-n_{0}\right\}<\frac{1}{\bar{n}}
$$

With the choice (A19) for $n_{0}$, therefore, the upper bound for the right-hand side of (A16) may be written as

$$
\begin{aligned}
2 \sum\left|C_{n}\right|^{2} \sin ^{2}\left(\phi_{1} / 2\right) & \leq 2 \sum_{n=0}^{n_{0}}\left|C_{n}\right|^{2}+\frac{1}{2} \sum_{n=n_{0}}^{\infty}\left|C_{n}\right|^{2} \phi_{1}^{2} \\
& <\frac{2}{\bar{n}}+\frac{g^{2} t^{2}}{8 \bar{n}} \sum_{n=n_{0}}^{\infty}\left|C_{n}\right|^{2} \frac{(\bar{n}-n)^{2}}{(\sqrt{n+1}+\sqrt{n})^{2}}\left|\frac{1}{\sqrt{\bar{n}}+\sqrt{n}}+\frac{1}{\sqrt{\bar{n}}+\sqrt{n+1}}\right|^{2}
\end{aligned}
$$

[where the definition (A13a) for $\phi_{1}$ has been used]. An upper bound for the last term in readily obtained by replacing the term in large parentheses (squared) by $1 / 4 \bar{n}$ and noting that $1 /(\sqrt{n+1}+\sqrt{n})^{2}<1 / 4 n_{0}$ when $n$ runs from $n_{0}$ to infinity; after which, the summation may be extended down to $n=0$ again: 


$$
\begin{aligned}
\frac{g^{2} t^{2}}{8 \bar{n}} \sum_{n=n_{0}}^{\infty}\left|C_{n}\right|^{2} \frac{(\bar{n}-n)^{2}}{(\sqrt{n+1}+\sqrt{n})^{2}} & \left(\frac{1}{\sqrt{\bar{n}}+\sqrt{n}}+\frac{1}{\sqrt{\bar{n}}+\sqrt{n+1}}\right)^{2} \\
& <\frac{g^{2} t^{2}}{128 n_{0} \bar{n}^{2}} \sum_{n=n_{0}}^{\infty}\left|C_{n}\right|^{2}(\bar{n}-n)^{2}<\frac{g^{2} t^{2}}{128 n_{0} \bar{n}^{2}} \sum_{n=0}^{\infty}\left|C_{n}\right|^{2}(\bar{n}-n)^{2}=\frac{g^{2} t^{2}}{128 n_{0} \bar{n}}
\end{aligned}
$$

This term, therefore, goes to zero as $\bar{n} \rightarrow \infty$, for all $t$; again, one may contemplate the limit $t \rightarrow \infty$, provided that $t$ go to infinity more slowly than $\left(n_{0} \bar{n}\right)^{1 / 2}$. Note that, by Eq. (A19), $n_{0}$ goes to infinity essentially as $\bar{n}$ itself, so that even if $t$ goes to infinity as $n_{0}^{p}$, where $p<1$, this term will be negligible for $n_{0}$ sufficiently large.

In conclusion, then, in the limit $\bar{n} \rightarrow \infty$, the whole term in square brackets in Eq. (A14) may be replaced just by unity, and therefore the evolution of the state $|+\rangle|v\rangle$ is just given by

$$
\begin{aligned}
\left.|+\rangle|v\rangle\right|_{t=0} \rightarrow & \frac{1}{\sqrt{2}}\left(e^{-i \phi} e^{-i g t / 2 \sqrt{\bar{n}}}|a\rangle+|b\rangle\right) \\
& \times \sum_{n=0}^{\infty} C_{n} e^{-i g t \sqrt{n}}|n\rangle,
\end{aligned}
$$

which shows that in this limit the states of both the atom and the field remain pure throughout. It is easy to verify that one has analogously, for the state

$$
\begin{aligned}
&|-\rangle=\frac{1}{\sqrt{2}}\left(e^{-i \phi}|a\rangle-|b\rangle\right) \\
&\left.|-\rangle|v\rangle\right|_{t=0} \rightarrow \frac{1}{\sqrt{2}}\left(e^{-i \phi} e^{i g t / 2 \sqrt{\bar{n}}}|a\rangle-|b\rangle\right) \\
& \times \sum_{n=0}^{\infty} C_{n} e^{i g t \sqrt{n}}|n\rangle .
\end{aligned}
$$

\section{APPENDIX B: SOME PROPERTIES OF THE ASYMPTOTIC FIELD STATES}

As discussed in the text, and shown in Appendix A, the asymptotic field states

$$
\left|\Phi_{ \pm}(t)\right\rangle=e^{-\bar{n} / 2} \sum_{n=0}^{\infty} \frac{\bar{n}^{n / 2}}{\sqrt{n !}} e^{-i n \phi} e^{\mp i g t \sqrt{n}}|n\rangle
$$

[Eq. (7) of this paper] represent the field in the JCM in the limit as $\bar{n} \rightarrow \infty$. As already mentioned in Sec. II, these states will yield results for the expectation values of field operators which are correct to leading order in $\bar{n}$; however, in Appendix A it was only possible to prove the asymptotic results (A24) and (A25) up to terms whose norm squared might go to zero only as fast as $1 / \bar{n}$, which means that it is entirely possible that the expectation values calculated using the states $\left|\Phi_{+}(t)\right\rangle$ might not be accurate beyond the leading order. In particular, the terms neglected in Appendix A might quite possibly make a finite contribution to an expectation value such as $\left\langle a^{2}\right\rangle$ (which to leading order is expected to grow like $\bar{n}$ as $\bar{n} \rightarrow \infty)$.

This means that it is possible that the states $\left|\Phi_{ \pm}(t)\right\rangle$ would not yield an accurate result for something like the degree of squeezing in the field, since that quantity involves the difference $\left\langle a^{2}\right\rangle-\langle a\rangle^{2}$, in which (if there is to be any squeezing at all) the leading-order term in $\bar{n}$ must cancel. It is of course also possible that there are further cancellations among the neglected terms which might make this result, calculated using the states $\left|\Phi_{ \pm}(t)\right\rangle$, more accurate than, based on these considerations, one would appear to have a right to expect. In any case, at present it is not possible to claim that all the results to be derived for the states $\left|\Phi_{ \pm}(t)\right\rangle$ in this appendix are directly relevant to the JCM, which is in fact the reason why these results are relegated to the appendix instead of being part of the main text. But these are states of the quantized field that are interesting in their own right and which do not appear to have been studied previously, and people have published papers for less than this.

The first obvious thing about the states (B1) is that their photon-number distribution is exactly Poissonian, since their coefficients in the number state basis are identical to those of the coherent states except for a phase factor. In fact, the states (B1) are a special example of a general class of states known as generalized coherent states [33] and characterized by this property. Thus we have

$$
\left\langle\Delta^{2} n\right\rangle=\langle n\rangle=\bar{n} .
$$

The coherent state approximation to the states (B1) has also been mentioned in the text [Sec. III, Eq. (15)]. For short times, $t<<\sqrt{\bar{n}} / g$, one has

$$
\left|\Phi_{ \pm}(t)\right\rangle \simeq e^{\mp i g t \sqrt{\bar{n}} / 2}\left|v e^{\mp i g t / 2 \sqrt{\bar{n}}}\right\rangle
$$

where $v=\sqrt{\bar{n}} e^{-i \phi}$, and the notation on the right-hand side means a coherent state with the complex amplitude shown. This shows that the states $\left|\Phi_{ \pm}(t)\right\rangle$ evolve in a circle in phase space, keeping a constant amplitude while the phase grows as $\phi_{ \pm}= \pm g t / 2 \sqrt{\bar{n}}$.

For longer times, as discussed in Sec. IV, the approximation (B3) breaks down and the quadratic terms in the expansion of the square root of $n$ about $\bar{n}$ have to be taken into account. For large $\bar{n}$, this yields the next-order approximation,

$$
\begin{aligned}
\left|\Phi_{ \pm}(t)\right\rangle \simeq e^{\mp i g t \sqrt{\bar{n}} / 2} e^{-\bar{n} / 2} \\
\times \sum_{n=0}^{\infty} \frac{\bar{n}^{n / 2}}{\sqrt{n !}} e^{-i n(\phi \pm g t / 2 \sqrt{\bar{n}})} \\
\quad \times e^{ \pm i(n-\bar{n})^{2} g t / 8 \bar{n}^{3 / 2}}
\end{aligned}
$$


which is good provided $t<<\bar{n}^{3 / 2} / g$; in fact, it may be expected to hold asymptotically even for $t \rightarrow \infty$ provided gt $/ \sqrt{\bar{n}}$ remains finite. Since the largest JCM time scale considered in this paper is the revival time scale given by $t_{R}=2 \pi \sqrt{\bar{n}} / g$, this approximation (B4) may be useful for most practical purposes, in the limit of large $\bar{n}$, even over a large number of revivals.

The state (B4) is no longer as simple as a coherent state. A calculation of $\left\langle a^{p}\right\rangle(p \geq 1)$ based on (B4) yields

$$
\begin{aligned}
\left\langle a^{p}\right\rangle_{ \pm} \simeq & e^{-\bar{n}} e^{-i p \phi} \bar{n}^{p / 2} e^{\mp i p g t / 2 \sqrt{\bar{n}}} \\
& \times \sum_{n=0}^{\infty} \frac{\bar{n}^{n}}{n !} \exp \left\{ \pm i\left[2 p(n-\bar{n})+p^{2}\right] g t / 8 \bar{n}^{3 / 2}\right\} \\
\simeq & e^{-i p \phi_{\bar{n}} p / 2} e^{\mp i p g t / 2 \sqrt{\bar{n}}} \\
& \times\left[1 \pm i \frac{p^{2}}{4 \bar{n}}\left[\frac{g t}{2 \sqrt{\bar{n}}}\right]-\frac{p^{2}}{8 \bar{n}}\left[\frac{g t}{2 \sqrt{\bar{n}}}\right]^{2}\right],
\end{aligned}
$$

where the terms kept inside the square brackets go to zero, for $g t / \sqrt{\bar{n}}$ finite, as $1 / \bar{n}$, but clearly result in an overall finite contribution to $\left\langle a^{p}\right\rangle$ for $p \geq 2$, and to $\langle a\rangle^{2}$, because of the factor of $\bar{n}^{p / 2}$.

The leading term in $\bar{n}$ in Eq. (B5) is in fact identical to what one would have calculated based on the coherent state approximation (B3), which reinforces the interpretation of the state $\left|\Phi_{ \pm}(t)\right\rangle$ as a "quasicoherent" one having amplitude $\sqrt{\bar{n}}$ and phase $\phi \pm g t / 2 \sqrt{\bar{n}}$ (the convention used here is that a "positive" optical field phase $\phi$ corresponds to a phasor like $\left.e^{-i \phi}\right)$. The smaller terms in square brackets are "squeezing" terms, and, as a matter of fact, they are incorrect as given in Eq. (B5) (which nicely illustrates, in fact, the danger of relying on asymptotic expressions to provide much information beyond the leading order); that is, the approximation (B4) used to derive (B5) already predicts the wrong magnitude for these correction terms, when compared with a more accurate expansion based directly on (B1), as will be shown present [see Eq. (B8), below]. Nonetheless, somewhat remarkably, due to a cancellation the squeezing predicted from (B5) agrees with the result of the more accurate calculation.

This more accurate calculation of $\left\langle a^{p}\right\rangle$ proceeds as follows. Let $\phi=0$ to simplify the equations and also, for typographical convenience, concentrate on the state $\left|\Phi_{+}(t)\right\rangle$. We have

$$
\left\langle a^{p}\right\rangle_{+}=e^{-\bar{n}} \bar{n} p / 2 \sum_{n=0}^{\infty} \frac{\bar{n}^{n}}{n !} e^{-i g t(\sqrt{n+} p-\sqrt{n})}
$$

where, to the desired accuracy,

$$
\begin{aligned}
\sqrt{n+p}-\sqrt{n} \simeq & \frac{p}{2 \sqrt{\bar{n}}}-\frac{p^{2}}{8 \bar{n}^{3 / 2}}-\frac{p}{4 \bar{n}^{3 / 2}}(n-\bar{n}) \\
& +\frac{3 p}{16 \bar{n}^{5 / 2}}(n-\bar{n})^{2} .
\end{aligned}
$$

The guiding principle here is that $(n-\bar{n})$ is of the order of $\sqrt{\bar{n}}$ and $t$ is of the order of $\sqrt{\bar{n}} / g$ (or possibly smaller, of course). The terms kept in (B7) are those which when multiplied by $g t$ do not go to zero faster than $1 / \bar{n}$.

Substituting (B7) into (B6) and expanding the exponent in powers of the small terms yields the result

$$
\begin{aligned}
\left\langle a^{p}\right\rangle_{+} \simeq \bar{n}^{p / 2} e^{-i p g t / 2 \sqrt{\bar{n}}} & 1+i \frac{p^{2}}{4 \bar{n}} \frac{g t}{2 \sqrt{\bar{n}}}-i \frac{3 p}{8 \bar{n}} \frac{g t}{2 \sqrt{\bar{n}}} \\
& \left.-\frac{p^{2}}{8 \bar{n}}\left[\frac{g t}{2 \sqrt{\bar{n}}}\right]^{2}\right] \cdot \quad \text { (B8) }
\end{aligned}
$$

The result for $\left|\Phi_{-}(t)\right\rangle$ is obtained from (B8), formally, by changing the sign of $t$. Equation (B8) is seen to differ from the approximate result (B5) by a term proportional to $p$ which, to the order considered, cancels out when the difference $\left\langle a^{2}\right\rangle-\langle a\rangle^{2}$ is taken. Hence, either (B5) or (B8) may be used to calculate the squeezing.

Squeezing [31] may be defined rather generally in terms of the (Hermitian) quadrature operator $a_{\theta}$,

$$
a_{\theta}=\frac{1}{2}\left(a e^{-i \theta}+a^{\dagger} e^{i \theta}\right)
$$

For a coherent state the variance of $a_{\theta}$ is equal to $\frac{1}{4}$ for all $\theta$. A state is said to be squeezed if there is some angle $\theta$ for which $\left\langle\Delta^{2} a_{\theta}\right\rangle<\frac{1}{4}$. In general, for any angle $\theta$ one must have $\left\langle\Delta^{2} a_{\theta}\right\rangle\left\langle\Delta^{2} a_{\theta+\pi / 2}\right\rangle \geq \frac{1}{16}$; minimumuncertainty squeezed states are those for which this relationship holds with an equal sign

One has in general

$$
\begin{aligned}
\left\langle\Delta^{2} a_{\theta}\right\rangle \equiv\left\langle a_{\theta}^{2}\right\rangle & -\left\langle a_{\theta}\right\rangle^{2} \\
=\frac{1}{4}+\frac{1}{4}\left[\left(\left\langle a^{2}\right\rangle-\langle a\rangle^{2}\right) e^{-2 i \theta}\right. & \\
& \left.\quad+\left\langle a^{\dagger} a\right\rangle-|\langle a\rangle|^{2}+\text { c.c. }\right]
\end{aligned}
$$

and therefore, using (B8) [with (B2) for $\left\langle a^{\dagger} a\right\rangle \equiv\langle n\rangle$ ],

$$
\begin{aligned}
\left\langle\Delta^{2} a_{\theta}\right\rangle_{+} & =\frac{1}{4}+\frac{1}{4}\left[\frac{(g t)^{2}}{8 \bar{n}}-\frac{(g t)^{2}}{8 \bar{n}} \cos \left[\frac{g t}{\sqrt{\bar{n}}}+2 \theta\right]+\frac{g t}{2 \sqrt{\bar{n}}} \sin \left[\frac{g t}{\sqrt{\bar{n}}}+2 \theta\right]\right] \\
& =\frac{1}{4}+\frac{1}{4}\left[\frac{(g t)^{2}}{8 \bar{n}}-\left[\frac{(g t)^{2}}{4 \bar{n}}+\frac{(g t)^{4}}{64 \bar{n}^{2}}\right]^{1 / 2} \cos \left[\frac{g t}{\sqrt{\bar{n}}}+2 \theta+\theta_{0}\right)\right],
\end{aligned}
$$


where the angle $\theta_{0}$ is defined by

$$
\cot \theta_{0}=\frac{g t}{4 \sqrt{\bar{n}}} \text {. }
$$

Equation (B11) shows that, for all times $t>0$, the states $\left|\Phi_{ \pm}(t)\right\rangle$ are, in fact, squeezed. The direction of maximum squeezing corresponds to $\theta=-g t / 2 \sqrt{\bar{n}}-\theta_{0} / 2$ for the state $\left|\Phi_{+}(t)\right\rangle$, and to $\theta=g t / 2 \sqrt{\bar{n}}+\theta_{0} / 2$ for the state $\left|\Phi_{-}(t)\right\rangle$. As $t$ grows, this direction is more and more along the direction of the average field $\langle a\rangle$, i.e., $\theta_{0} \rightarrow 0$ for large $t$ [recall that $\langle a\rangle_{ \pm}$goes as $\exp (\mp i g t / 2 \sqrt{\bar{n}})]$; this means that the states become squeezed in amplitude, but one must remember that the actual intensity fluctuations, given by (B2), always have their Poissonian value $\bar{n}$, in spite of the squeezing going on. In this important respect these states differ from the crescent squeezed states of Ref. [14] (which are squeezed in photon number) in spite of their formal resemblance.

The maximum squeezing grows (i.e., $\left\langle\Delta^{2} a_{\theta}\right\rangle$ decreases) as $t$ grows. In fact,

$$
\left\langle\Delta^{2} a_{\theta}\right\rangle_{\min }=\frac{1}{4}+\frac{1}{4}\left[\frac{(g t)^{2}}{8 \bar{n}}-\left[\frac{(g t)^{2}}{4 \bar{n}}+\frac{(g t)^{4}}{64 \bar{n}^{2}}\right]^{1 / 2}\right]
$$

goes to zero for large $t$.

These features are illustrated by the contour plots in Fig. 1. Note that the fact that the states $\left|\Phi_{ \pm}(t)\right\rangle$ are at all times squeezed indicates that their Glauber $P$ representation [7] is probably singular (at the very least, it must be non-positive definite). A convenient alternative is provided by the $Q$ function defined in the text [Eq. (30)]. Figure 1 shows contour plots for the $Q$ function calculated using the approximation (B4). We have

$$
\begin{aligned}
Q_{+}(\alpha, t) & =\left|\left\langle\alpha \mid \Phi_{+}(t)\right\rangle\right|^{2} \\
& \simeq e^{-\bar{n}-r^{2}}\left|\sum_{o}^{\infty} \frac{(r \sqrt{\bar{n}})^{n}}{n !} e^{-i n(g t \sqrt{\bar{n}} / 2-\phi)+i g t(n-\bar{n})^{2} / 8 \bar{n}^{3 / 2}}\right|^{2},
\end{aligned}
$$

where $\alpha=r e^{i \phi}$ (this angle $\phi$ is a variable, not to be mistaken for the initial field phase $\phi$ used elsewhere in the paper; that phase has been set equal to zero for convenience in what follows). The sum may be approximated by an integral for large $\bar{n}$, using the Gaussian approximation to a Poisson distribution,

$$
\frac{(r \sqrt{\bar{n}})^{n}}{n !} \simeq e^{r^{2} \bar{n}} \frac{1}{\sqrt{2 \pi r \sqrt{\bar{n}}}} e^{-(n-r \sqrt{\bar{n}})^{2} / 2 r \sqrt{\bar{n}}} .
$$

This yields the simple result

$$
Q_{+}(r, \phi, t) \simeq \frac{1}{\sqrt{s(t)}} e^{-(r-\sqrt{\bar{n}})^{2}} e^{-r \sqrt{\bar{n}}\left[\phi-\phi_{0}(t)\right]^{2} / s(t)},
$$

where

$$
\phi_{0}(t) \equiv-\frac{3 g t}{4 \sqrt{\bar{n}}}+\frac{r g t}{4 \bar{n}}
$$

and

$$
s(t) \equiv 1+\left(\frac{r g t}{4 \bar{n}}\right)^{2} .
$$

(For $Q_{-}$, merely reverse the sign of $t$.) This is a better approximation to the actual state of the field in the JCM than the lowest-order approximation of Eiselt and Risken [Eq. (A8) of Ref. [16]], whose contour lines are just ellipses; the contour lines of (B16), plotted in Fig. 1, appear to be very close to the ones calculated numerically by Eiselt and Risken and plotted in their Fig. 1(a).

The states $\left|\Phi_{ \pm}(t)\right\rangle$ are the states of the field in the JCM when the initial atomic state is $| \pm\rangle$, respectively. If the initial atomic state is instead a superposition of $|+\rangle$ and $|-\rangle$ (such as, e.g., the energy eigenstates $|a\rangle$ and $|b\rangle)$, the field state is in general a mixture of $\left|\Phi_{+}(t)\right\rangle$ and $\left|\Phi_{-}(t)\right\rangle$. Such a mixture will not in general be a pure state, nor will it in general be a squeezed state because the phases of $\left|\Phi_{+}(t)\right\rangle$ and $\left|\Phi_{-}(t)\right\rangle$, as has been shown, evolve in opposite directions, so that at almost any time $t>0$ the spread (uncertainty) in the field real and imaginary parts, relative to any axes, is very large. At halfrevival time $[t=(2 q+1) \pi \sqrt{\bar{n}} / g, q=0,1, \ldots]$, the field's state is pure, a coherent superposition of $\left|\Phi_{+}(t)\right\rangle$ and $\left|\Phi_{-}(t)\right\rangle$; these are macroscopic fields $180^{\circ}$ out of phase, and hence the total state of the field shows no squeezing at all (in fact, it shows the maximum spread for $\left\langle\Delta^{2} a_{\theta}\right\rangle$, of the order, as Fig. 1 illustrates, of $\bar{n}$ itself), because of the uncertainty in the direction in which the phasor is pointing (straight up or straight down?), in spite of the fact that the states being superposed are squeezed.

The situation is different at the revival times $(t=2 q \pi \sqrt{\bar{n}} / g, q=0,1, \ldots)$, since at these times the states $\left|\Phi_{+}(t)\right\rangle$ and $\left|\Phi_{-}(t)\right\rangle$ overlap appreciably, i.e., their $Q$ functions are concentrated to a large extent in the same region of the phase space, and moreover, according to (B11) and (B12), they are both squeezed along more or less the same direction (as the angle $\theta_{0}$ decreases with time). Hence one expects the total field to show squeezing at this point [note in passing that, if the initial state is one of the energy eigenstates, the field at the revival time is not a coherent superposition, but an incoherent mixture of $\left|\Phi_{+}(t)\right\rangle$ and $\left.\left|\Phi_{-}(t)\right\rangle\right]$. In fact, since the squeezing, as (B13) indicates, increases with time, and the angle $\theta_{0}$ approaches zero, one expects greater and greater squeezing for successive revivals.

These predictions are at least in qualitative agreement 
with the results of Kuklinski and Madajczyk [6] for squeezing in the JCM; which is perhaps more than one had a right to expect, given the reservations expressed at the beginning of this appendix on the reliability of a calculation of squeezing in the JCM based on the asymptotic states $\left|\Phi_{+}(t)\right\rangle$ and $\left|\Phi_{-}(t)\right\rangle$. Doubtless these states do provide a good approximation to the field in the JCM; it may be worth pursuing the question of to what extent they may be used as a first step for a quantitatively accurate calculation of the squeezing in the JCM.

Finally, to return to the properties of the states $\left|\Phi_{+}(t)\right\rangle$ and $\left|\Phi_{-}(t)\right\rangle$ themselves, it is worth mentioning the connection between the approximation (B4), which leads to the curves in Fig. 1, and the "number-phase minimum-uncertainty states" or "crescent states" investigated by Yamamoto and co-workers [13,14]; indeed, Eq. (B14) is essentially equivalent to Eq. (3.17) of the paper of Kitagawa and Yamamoto [14], which concerned a state generated in a nonlinear Kerr-type medium. A study of $Q(r, \phi, t)$ for long times shows that the distribution eventually does adopt a nicely shaped, almost symmetric, crescent form. The main difference (and a major one) is that the states $\left|\Phi_{+}(t)\right\rangle$ and $\left|\Phi_{-}(t)\right\rangle$, according to Eq. (B2), never exhibit any photon-number squeezing. This is probably due to the fact that for the states $\left|\Phi_{+}(t)\right\rangle$ and $\left|\Phi_{-}(t)\right\rangle$ the axis for squeezing and the direction of the coherent amplitude are tilted at a different angle, in phase space, than for the states of Ref. [14].
[1] E. T. Jaynes and F. W. Cummings, Proc. IEEE 51, 89 (1963).

[2] For a review, see H. Walther, Phys. Scr. T23, 165 (1988); F. Diedrich, J. Krause, G. Rempe, M. O. Scully, and H. Walther, IEEE J. Quantum Electron. QE-24, 1314 (1988). More recent results are reported in G. Rempe, F. Schmidt-Kaler, and H. Walther, Phys. Rev. Lett. 64, 2783 (1990).

[3] J. H. Eberly, N. B. Narozhny, and J. J. SánchezMondragón, Phys. Rev. Lett. 44, 1323 (1980); N. B. Narozhny, J. J. Sánchez-Mondragón, and J. H. Eberly, Phys. Rev. A 23, 236 (1981).

[4] For a review and references to original literature see H. I. Yoo and J. H. Eberly, Phys. Rep. 118, 239 (1985).

[5] P. Meystre and M. S. Zubairy, Phys. Lett. 89A, 390 (1982).

[6] J. R. Kuliński and J. L. Madajczyk, Phys. Rev. A 37, 3175 (1988).

[7] Coherent states were first introduced by E. Schrödinger, Naturwissenschaften 14, 664 (1926), as the classical states of a quantum harmonic oscillator. Their introduction in quantum optics is due to R. J. Glauber, Phys. Rev. 131, 2766 (1963).

[8] Studies dealing with some of these questions in the context of the micromaser include J. Krause, M. O. Scully, T. Walther, and H. Walther, Phys. Rev. A 39, 1915 (1989); M. O. Scully and H. Walther, ibid. 39, 5229 (1989); P. Filipowicz, J. Javanainen, and P. Meystre, J. Opt. Soc. Am. B 3, 906 (1986); and the papers in Ref. [9] below.

[9] J. J. Slosser, P. Meystre, and S. L. Braunstein, Phys. Rev. Lett. 63, 934 (1989); J. J. Slosser, P. Meystre, and E. M. Wright, Opt. Lett. 15, 233 (1990); J. J. Slosser and P. Meystre, Phys. Rev. A 41, 3867 (1990).

[10] S. J. D. Phoenix and P. L. Knight, Ann. Phys. (N.Y.) 186, 381 (1988).

[11] No attempt will be made here to establish a detailed connection between this result and those to be presented in this paper; see, for more details, S. J. D. Phoenix and P. L. Knight, this issue, Phys. Rev. A 44, 6023 (1991); Phys. Rev. Lett. 66, 2833 (1991).

[12] J. Gea-Banacloche, Phys. Rev. Lett. 65, 3385 (1990).

[13] Y. Yamamoto, N. Imoto, and S. Machida, Phys. Rev. A 33, 3243 (1986).

[14] M. Kitagawa and Y. Yamamoto, Phys. Rev. A 34, 3974 (1986).
[15] J. Eiselt and H. Risken, Opt. Commun. 72, 351 (1989).

[16] J. Eiselt and H. Risken, Phys. Rev. A 43, 346 (1991).

[17] The phase distribution of the field has been calculated by Ho Trung Dung, R. Tanaś, and A. S. Shumovsky, Opt. Commun. 79, 462 (1990).

[18] A similar result was obtained for the so-called Ramancoupled model by S. J. D. Phoenix and P. L. Knight, J. Opt. Soc. Am. B 7, 116 (1990).

[19] B. Yurke and D. Stoler, Phys. Rev. Lett. 57, 13 (1986).

[20] R. R. Puri and G. S. Agarwal, Phys. Rev. A 35, 3433 (1987).

[21] G. S. Agarwal and R. R. Puri, J. Opt. Soc. Am. B 5, 1669 (1988).

[22] K. Zaheer and M. S. Zubairy, Phys. Rev. A 39, 2000 (1989).

[23] J. I. Cirac and L. L. Sánchez-Soto, Phys. Rev. A 42, 2851 (1990).

[24] K. Zaheer and M. S. Zubairy, Phys. Rev. A 37, 1628 (1988).

[25] A. Aspect, P. Grangier, and G. Roger, Phys. Rev. Lett. 49, 91 (1982).

[26] For a treatment of shot noise in photodetection, see, e.g., R. H. Kingston, Detection of Optical and Infrared Radiation (Springer-Verlag, Berlin, 1978). According to conventional treatments, photocurrent shot noise limits phase detection because a phase change is always detected through the measurement of an intensity change (e.g., in an interferometer). Shot noise is usually written as proportional to $(B / P)^{1 / 2}$, where $B$ is detector bandwidth and $P$ is power in the light beam; if $B=1 / \Delta t$, where $\Delta t$ is the measurement duration, $P \Delta t=\hbar \omega \bar{n}$, where $\bar{n}$ is the number of photons available. For an example of an application of the conventional results to the problem of phase detection, see R. L. Forward, Phys. Rev. D 17, 379 (1978). Numerical factors on the right-hand side of Eq. (21) may vary somewhat depending on the experimental arrangement.

[27] W. H. Zurek, Phys. Rev. D 26, 1862 (1982).

[28] P. Pfeifer, Helv. Phys. Acta 53, 410 (1980); P. Pfeifer, in Advances in Chemical Physics, edited by I. Prigogine and S. A. Rice (Wiley, New York, 1983), Vol. 53, p. 341. Not directly related to measurement, but involving a superselection rule similar to the one derived later in this section: P. Pfeifer, Phys. Rev. A 26, 701 (1982). 
[29] G. J. Milburn, Opt. Acta 31, 671 (1984).

[30] For a simple explanation of this point, see, e.g., J. GeaBanacloche and G. Leuchs, J. Mod. Opt. 34, 793 (1987) [following C. M. Caves, Phys. Rev. D 23, 1693 (1981)]. This has recently been established formally by S. M. Barnett and D. T. Pegg, J. Mod. Opt. 36, 7 (1989), using their Hermitian phase operator.

[31] A good, relatively concise introduction to squeezed states has been provided by R. Loudon and P. L. Knight, J. Mod. Opt. 34, 709 (1987). A longer, very thorough review is that of K. Zaheer and M. S. Zubairy, in Advances in Atomic, Molecular and Optical Physics, edited by Sir D. Bates and B. Bederson (Academic, New York, 1991), Vol. 28 , p. 143.

[32] Again, a formal proof of a relationship like (22) (except for a numerical factor) has been provided by J. A. Vaccaro and D. T. Pegg, J. Mod. Opt. 37, 17 (1990). Simpler, heuristic arguments may be found in the first paper quoted in Ref. [30].

[33] U. Titulaer and R. Glauber, Phys. Rev. 145, 1041 (1965); Z. Byalinicka-Birula, ibid. 173, 1207 (1968); D. Stoler, Phys. Rev. D 4, 2309 (1971).

[34] E. Schrödinger, Naturwissenschaften 23, 812 (1935). An
English translation of this article may be found in the book Quantum Theory and Measurement, edited by J. A. Wheeler and W. H. Zurek (Princeton University Press, Princeton, NJ, 1983), p. 152.

[35] G. J. Milburn, Phys. Rev. A 33, 674 (1986).

[36] S. M. Barnett and P. L. Knight, Phys. Rev. A 33, 2444 (1986); also of interest is the comparison between the effects of losses on revivals and on squeezing, in Ref. [6], see also Ref. [16].

[37] A. O. Caldeira and A. J. Leggett, Phys. Rev. A 31, 1059 (1985); G. J. Milburn and C. A. Holmes, Phys. Rev. Lett. 56, 2237 (1986).

[38] P. L. Knight, Phys. Scr. T12, 51 (1986).

[39] It falls outside the scope of this paper to provide full references for all these variations on the JCM. Many references may be found in the paper by Lin-sheng $\mathrm{He}, \mathrm{J}$. Opt. Soc. Am. B 6, 1915 (1989).

[40] B. Buck and C. V. Sukumar, Phys. Lett. 81A, 132 (1981).

[41] Handbook of Mathematical Functions, Natl. Bur. Stand. Appl. Math. Ser. No. 55, edited by M. Abramowitz and I. A. Stegun (U.S. GPO, Washington, DC, 1965), Eq. 26.1.41. 